\title{
DEGREE 1 CURVES IN THE DWORK PENCIL AND THE MIRROR QUINTIC
}

\author{
ANCA MUSTAŢ Ǎ
}

\begin{abstract}
We give a description of the relative Hilbert scheme of lines in the Dwork pencil of quintic threefolds. We describe the corresponding relative Hilbert scheme associated to the mirror family of quintic threefolds.
\end{abstract}

\section{INTRODUCTION}

0.1. The relative Hilbert scheme of lines in the Dwork family of quintics. This paper takes a look at a very well known family of CalabiYau threefolds: the family $\rho: \mathcal{X} \rightarrow \mathbb{C}$ whose fiber $X_{t}$ over $t \in \mathbb{C}$ is given in $\mathbb{P}^{4}$ by the equation $\left(F_{t}=0\right)$, where

$$
F_{t}\left(z_{0}: z_{1}: z_{2}: z_{3}: z_{4}\right)=z_{0}^{5}+z_{1}^{5}+z_{2}^{5}+z_{3}^{5}+z_{4}^{5}-5 t z_{0} z_{1} z_{2} z_{3} z_{4} .
$$

This family is known as the Dwork pencil of quintics.

We will study the relative Hilbert Scheme of lines in the family $\mathcal{X}$. Let $\pi: \mathcal{H} \rightarrow \mathbb{C}$ denote this relative scheme. We show that the structure of $\mathcal{H}$ is

$$
\mathcal{H}=\mathcal{S} \cup \bigcup_{i=1}^{375} Z_{i}
$$

where $\mathcal{S}$ is a smooth irreducible surface, proper over $\mathbb{C}$ and for each $i$, $\pi_{\mid Z_{i}}: Z_{i} \rightarrow \mathbb{C}$ is an isomorphism.

The general fiber of $\pi_{\mid \mathcal{S}}: \mathcal{S} \rightarrow \mathbb{C}$ is a smooth proper curve consisting of two isomorphic components, each of genus 626 .

The Stein factorization of $\pi_{\mid \mathcal{S}}$ is

$$
\mathcal{S} \stackrel{\pi_{2}}{\longrightarrow} C_{2} \stackrel{\beta}{\longrightarrow} \mathbb{C}
$$

where $\beta$ extends to a double cover $\bar{\beta}: \bar{C}_{2} \rightarrow \overline{\mathbb{C}}=\mathbb{P}^{1}$ branched at six points: $t=0$ and the fifth roots of $\frac{2^{7}}{3}$.

Let $\tilde{G}(2,5)$ denote the blow-up of the Grassmannian $G(2,5)$ of lines in $\mathbb{P}^{4}$, along the subvarieties parametrizing lines inside the coordinate hyperplanes of $\mathbb{P}^{4}$. The closure $\tilde{\mathcal{S}}$ of $\mathcal{S}$ in $\tilde{G}(2,5) \times \mathbb{P}^{1}$ is a smooth projective surface admitting a 30-1 finite morphism to the Fermat surface

$$
z_{0}^{5}+z_{1}^{5}+z_{2}^{5}+z_{3}^{5}=0
$$

Date: October 29, 2018. 
in $\mathbb{P}^{3}$.

The fiber $\tilde{\mathcal{S}}_{\infty}$ is the stable limit of the family $\mathcal{S}$ at $t=\infty$ and consists of two connected components $\tilde{\mathcal{C}}^{\xi}$ and $\tilde{\mathcal{C}}^{\xi^{2}}$, which are isomorphic and reducible:

$$
\tilde{\mathcal{C}}^{\xi}=\bigcup_{i=0}^{4} \tilde{\mathcal{C}}_{i}^{\xi}
$$

with $\tilde{\mathcal{C}}_{i}^{\xi}$ smooth isomorphic curves of genus 76 , intersecting pairwise transversely at 25 points - a total of 500 points.

The "mirror" Hilbert scheme. The group

$$
\hat{G}=\left\{\left(\mu_{0}: \mu_{1}: \mu_{2}: \mu_{3}: \mu_{4}\right) / \mu_{i}^{5}=1\right\} /\left\{(\mu: \mu: \mu: \mu: \mu) / \mu^{5}=1\right\}
$$

acting on $\mathbb{P}^{4}$ also acts equivariantly on $\mathcal{S}, C_{2}$ and $\mathbb{C}$. The action on $\mathbb{C}$ is via $\mu=\prod_{j=0}^{4} \mu_{j}$. Putting parameter $w=t^{5}$ on $\mathbb{C} / \hat{G}$ we have the Stein factorization

$$
(\mathcal{S} / \hat{G}) \stackrel{\pi_{2} / \hat{G}}{\longrightarrow}\left(C_{2} / \hat{G}\right) \stackrel{\beta / \hat{G}}{\longrightarrow}(\mathbb{C} / \hat{G})
$$

of $\psi$ where $\beta / \hat{G}$ extends to a double cover of $\mathbb{P}^{1}$ branched at 0 and $\frac{2^{7}}{3}$.

The general fiber of $\pi_{2} / \hat{G}$ is a smooth hyperelliptic irreducible curve of genus 6 .

The compactification $\tilde{\mathcal{S}} / \hat{G}$ of $\mathcal{S} / \hat{G}$ is a smooth, irreducible, rational surface.

The stable limit at $w=\infty$ of the family $\mathcal{S} / \hat{G}$ is

$$
\tilde{\mathcal{S}}_{\infty} / \hat{G}=(\tilde{\mathcal{C}} \xi / \hat{G}) \bigcup\left(\tilde{\mathcal{C}}^{\xi^{2}} / \hat{G}\right)
$$

where $\tilde{\mathcal{C}}^{\xi} / \hat{G}$ is the union of 5 smooth rational curves intersecting pairwise transversely.

0.2. History of problem. For positive integers $m$ and $n$, the Fermat Variety $X_{m}^{n}$ is the hypersurface in $\mathbb{P}^{n}$ given by equation

$$
z_{0}^{m}+z_{1}^{m}+\ldots+z_{n}^{m}=0 .
$$

The Fermat varieties have long been subject to the attention of mathematicians: the Fermat curves have been intensely studied by number theorists while the Fermat hypersurfaces in general offered interesting examples in Hodge theory.

The Dwork pencil is known today mostly for its quotient by the action of the group $\hat{G}$ defined in part (0.1), which after desingularization gives the mirror to the family of quintic threefolds. Mirror symmetry arose in 1991, when Candelas, de la Ossa, Green and Parkes used that principle to predict invariants very closely related to the expected numbers of rational curves of any degree on a quintic threefold. In the same year, A.Albano and S. Katz gave a first description of the Hilbert scheme of lines on the Fermat quintic threefold $X_{0}$ (see [AK1]). They proved that all the lines in $X_{0}$ are 
actually contained in 50 cones over some plane quintics. They also studied the deformation theory of these lines for general deformations of $X_{0}$.

Then they initiated the study of the relative Hilbert scheme of the Dwork pencil of quintics (see AK2 ). Their work built on B. van Geemen's discovery of a set of 5000 special lines on each member $X_{t}$ of the family - since this number exceeded the known invariant of 2875 lines, this established the Dwork pencil as the only known nontrivial family of quintics having each a continuous family of rational curves.

Also in AK2, A.Albano and S.Katz presented another proof of the fact that the Hilbert scheme $\mathcal{H}$ of the Dwork pencil is two dimensional. Their method consists of describing the lines contained in some $X_{t}$ as lines in $\mathbb{P}^{4}$ meeting each component of $B$, where $B$ is the base locus of the family $\mathcal{X}$. Since $B=\bigcup_{i=0}^{4} B_{i}$ with

$$
B_{i}=\left\{\left(z_{0}: z_{1}: z_{2}: z_{3}: z_{4}\right) \in X_{0} / z_{i}=0, \sum z_{j}^{5}=0\right\}
$$

the Fermat surfaces in the hyperplanes $H_{i}=\left\{z_{i}=0\right\}$, they show that the lines meeting each component of $B$ are parametrized by a complete intersection surface $I$ in the Grassmannian $G(2,5)$, given in Plücker coordinates by the equations:

$$
\sum_{j=0}^{4} p_{i j}^{5}=0
$$

for $i \in\{1, \ldots, 4\}$. However, this surface has a rather large number of irreducible components, only one of which will correspond to the Hilbert scheme $\mathcal{H}$. For example, for each $B_{i j}=B_{i} \cap B_{j}$, there are components of $I$ made of lines meeting $B_{i j}$ and the other components $B_{k}$ of the base locus. In this paper we isolate the Hilbert scheme from these other components and describe it more precisely.

This approach of A.Albano and S.Katz has proven fruitful in studying the closure of $\mathcal{H}$ in $G(2,5) \times \mathbb{P}^{1}$ and its desingularization, as well as in computing the class of these lines in the cohomology ring of $G(2,5)$ - as seen in sections 8 and 9 , respectively.

On the other hand, for $d \geq 2$, little is known today about the degree $\mathrm{d}$ rational curves lying in the Dwork pencil and their images in the mirror family. Our hope is that some of the proprieties of the degree 1 Hilbert scheme described in this paper might prove useful in studying this problem.

0.3. Method of proof. In analyzing the degree 1 relative Hilbert scheme of $\rho$, a special role is played by those lines in $X_{t}, t \neq 0$, which contain a point two of whose coordinates are 0 . We call these lines van Geemen lines, due to their use by Bert van Geemen in [AK1 to give the first proof of the fact that the relative dimension of $\mathcal{H}=H_{i l b}(\mathcal{X} / \mathbb{C})$ is 1 . It turns out that the local behavior of $\mathcal{H}$ at the Van Geemen lines is geometrically understandable. On the other hand, if we remove the lines in $X_{0}$ and the van Geemen lines from the universal line $\mathcal{U}$, the resulting quasi-projective 
scheme $\mathcal{U}^{\prime}$ admits a simple and uniquely symmetric algebraic presentation giving a birational map to $\mathbb{P}^{3}$.

The main tool in linking these two viewpoints came from a recent result of Clemens in C1 for K-trivial threefolds. There, the local analytical Hilbert scheme of a smooth curve is described as a gradient variety.

0.4. Notations. Throughout this paper, $\mathbb{P}^{n}$ will denote the $\mathrm{n}$-dimensional complex projective space, $G(k+1, n+1)$ will denote the Grassmann variety of $k$-dimensional projective subspaces of $\mathbb{P}^{n}$.

The Dwork pencil of quintics is denoted by $\mathcal{X}$ and its relative Hilbert scheme of lines by $\mathcal{H}$.

$\hat{G}$ is the group

$$
\left\{\left(\mu_{0}: \mu_{1}: \mu_{2}: \mu_{3}: \mu_{4}\right) / \mu_{i}^{5}=1\right\} /\left\{(\mu: \mu: \mu: \mu: \mu) / \mu^{5}=1\right\}
$$

and $G$ is its subgroup

$$
\left\{\left(\mu_{0}: \mu_{1}: \mu_{2}: \mu_{3}: \mu_{4}\right) / \mu_{i}^{5}=1, \prod_{i=0}^{4} \mu_{i}=1\right\} /\left\{(\mu: \mu: \mu: \mu: \mu) / \mu^{5}=1\right\}
$$

0.5. Acknowledgments. This paper would not have existed without the generous support of my advisor, Herbert Clemens. I am grateful for his sharing of mathematical ideas, his patience and for the sense of enjoyment that resulted from working with him. I would also like to thank his family for their kindness and hospitality.

I would like to thank Alberto Albano, Sheldon Katz and Bert van Geemen, both for setting up this problem, and for their sharing insights at different stages of the project. Thank you Gianluca Pacienza for useful conversation and encouragement. Mike Stillman, by teaching me Macaulay2, has added an exciting aspect to this work. I am grateful to Aaron Bertram for his support during these years at the University of Utah. 


\subsection{Contents.}

Section 1. Hilbert scheme $\mathcal{H}_{0}$ of lines in the Fermat quintic threefold.

Section 2. The relative Hilbert scheme $\mathcal{H}$ in a neighborhood of $\mathcal{H}_{0}$ : the Hilbert scheme as a gradient scheme.

Section 3. The van Geemen lines.

Section 4. Algebraic description of the universal line $\mathcal{U}$ away from $\mathcal{U}_{0}$ and the van Geemen lines.

Section 5. The surface component $\mathcal{S}$ of $\mathcal{H}$.

Section 6. Properties of the fiber $(\mathcal{S} / \hat{G})_{w}$.

Section 7. Properties of the fiber $\mathcal{S}_{t}$.

Section 8. The stable limits $\tilde{\mathcal{S}}_{\infty}$ and $\tilde{\mathcal{S}}_{\infty} / \hat{G}$.

Section 9. The class $[\overline{\mathcal{S}}]$ on $G(2,5)$. 


\section{Hilbert scheme $\mathcal{H}_{0}$ of Lines in the Fermat quintic threefold}

In AK1, $\mathcal{H}_{0 r e d}$ is proven to be the union of 50 Fermat quintic plane curves meeting transversely in pairs at 375 points. We will start by recalling the description of the Hilbert scheme $\mathcal{H}_{0}$ as in [AK1 and [CK]:

Notation . Let $\mu$ be a complex number such that $\mu^{5}=1$.

Let

$$
B_{i}=\left\{\left(z_{0}: z_{1}: z_{2}: z_{3}: z_{4}\right) \in X_{0} / z_{i}=0, \sum z_{j}^{5}=0\right\},
$$

for $i \in\{0,1,2,3,4\}$.

Let $B_{i j}=B_{i} \bigcap B_{j}$ and $B_{i j k}=B_{i} \bigcap B_{j} \bigcap B_{k}$, for $i, j, k \in\{0,1,2,3,4\}$.

$\mathcal{B}=\bigcup_{i=0}^{4} B_{i}$ is the base locus of the pencil of quintic hypersurfaces $X_{t}$. Each $B_{i j}$ is a quintic curve in $\mathbb{P}^{2}$ and each $B_{i j k}$ is a set of 5 points in $\mathbb{P}^{1}$.

Here $i, j, k, l, h$ will stand for pairwise different indices from 0 to 4 .

For each pair $(i, j)$ with $i, j \in\{0,1,2,3,4\}$, the hyperplane $\left(z_{i}+\mu z_{j}=0\right)$ intersects $X_{0}$ in a cone $C_{i, j, \mu}$ over the curve $B_{i j}$, whose vertex is one of the five points of $B_{k h l}$. There are 50 such cones. Two such cones $C_{i, j, \mu}$ and $C_{k, l, \nu}$ intersect in a unique line iff $i, j, k, l$ are pairwise different. Thus by counting we get 375 lines of intersection: each cone contains 15 such special lines, each special line is at the intersection of exactly 2 cones. The fact that the only lines in the Fermat quintic $X_{0}$ are those lying in one of these cones is proven in AK1.

From a local computation it can be seen that the Hilbert scheme $\mathcal{H}_{0}$ is not reduced at any of its points ( see [CK]). We will denote by $l_{1}$ one of the 375 special points of $\mathcal{H}_{0}$ and by $l_{2}$ any point of $\mathcal{H}_{0}$ away from the crossings. Then $\mathcal{H}_{0}$ can be described locally analytically as

$$
\operatorname{Spec} \mathbb{C}[[x, y]] /\left(y^{2}\right)
$$

in a neighborhood of $l_{2}$ and as

$$
\operatorname{Spec} \mathbb{C}[[x, y]] /\left(x^{3} y^{2}, x^{2} y^{3}\right)
$$

in a neighborhood of $l_{1}$. H.Clemens and H.Kley in [CK recovered from this local structure the Gromow-Witten invariant for lines in quintic threefolds: namely, the local primary decomposition of the ideal $\left(x^{3} y^{2}, x^{2} y^{3}\right)$ :

$$
\left(x^{3} y^{2}, x^{2} y^{3}\right)=\left(x^{2}\right) \cap\left(y^{2}\right) \cap(x, y)^{5}
$$

gives the following computation for the Gromow-Witten invariant:

$$
50 \cdot 2 \cdot\left(2 g_{0}-2\right)+375 \cdot 5=2875,
$$

where $g_{0}=6$ stands for the genus of the plane quintic.

In the remainder of this section we will answer the question concerning smoothness of the relative Hilbert scheme at the points $l_{1}$ and $l_{2}$. We will see that the $l_{2}$ are smooth points of $\mathcal{H}$ while the $l_{1}$ are not. For this purpose we compute the normal bundles $\mathcal{N}_{l_{1} \backslash X_{0}}, \mathcal{N}_{l_{1} \backslash \mathcal{X}}, \mathcal{N}_{l_{2} \backslash X_{0}}$, and $\mathcal{N}_{l_{2} \backslash \mathcal{X}}$. Notice also that the lines $\left\{l_{1}\right\}$ are contained in the base locus of the family $\left(X_{t}\right)_{t \in \mathbb{C}}$, 
thus the normal bundle $\mathcal{N}_{l_{1} \backslash X_{t}}$ will also give us information on the structure of $\mathcal{H}$ around the corresponding points in the fiber $\mathcal{H}_{t}$.

For simplicity, we will consider $l_{1}$ to be given by $\phi_{1}: \mathbb{P}^{1} \rightarrow \mathbb{P}^{4}$

$$
\phi_{1}((\alpha: \beta)):=(\alpha: \beta:-\alpha:-\beta: 0)
$$

$l_{2}$ to be given by the morphism $\phi_{2}: \mathbb{P}^{1} \rightarrow \mathbb{P}^{4}$

$$
\phi_{2}((\alpha: \beta)):=(\alpha: \beta:-\alpha: a \beta: b \beta)
$$

with $a b \neq 0,1+a^{5}+b^{5}=0$.

Using the action of the group $G \times S_{5}$ we can see that our computations hold for any $l_{1}$ and $l_{2}$ in $\mathcal{H}_{0}$.

We will use the following lemma, which is a version of lemma 1.8 in CK] in our particular case:

Lemma 1.1. Let $\mathcal{U}$ represent the universal family of lines over $\mathcal{H}$.

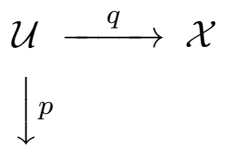

$\mathcal{H}$

Let $\mathcal{I}_{\mathcal{H}}$ denote the ideal sheaf of $\mathcal{H}$ in $G(2,5) \times \mathbb{C}$ and let d on $G(2,5) \times \mathbb{C}$ represent the differential in the direction of $G(2,5)$. Let $\mathcal{I}_{\mathcal{X}}$ denote the ideal sheaf of $\mathcal{X}$ in $\mathbb{P}^{4} \times \mathbb{C}, \mathcal{I}_{\mathcal{U}}$ the ideal sheaf of $\mathcal{U}$ in $\mathcal{H} \times \mathbb{C} \mathcal{X}$ and $\mathcal{J}_{\mathcal{U}}$ the ideal sheaf of $\mathcal{U}$ in $\mathcal{H} \times \mathbb{C}\left(\mathbb{P}^{4} \times \mathbb{C}\right)$. We will denote by $\omega$ the relative sheaf of differentials of $\mathcal{U}$ over $\mathcal{H}$. Then there is a commutative diagram:

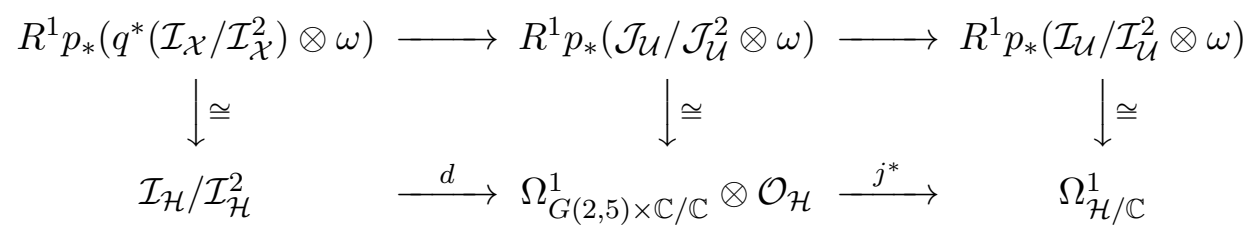

with $j^{*}$ surjective.

On fibers over $\{l\} \in \mathcal{H}_{t}$, the upper morphism in the previous sequence is: $H^{1}\left(\left.\mathcal{N}_{X_{t} \backslash \mathbb{P}^{4}}^{\vee}\right|_{l} \otimes \mathcal{O}_{l}(-2)\right) \rightarrow H^{1}\left(\mathcal{N}_{l \backslash \mathbb{P}^{4}}^{\vee} \otimes \mathcal{O}_{l}(-2)\right) \rightarrow H^{1}\left(\mathcal{N}_{l \backslash X_{t}}^{\vee} \otimes \mathcal{O}_{l}(-2)\right)$ which can be thought of, via Serre duality, as

$$
\left.\left.H^{0}\left(\mathcal{N}_{X_{t} \backslash \mathbb{P}^{4}}\right)\right|_{l}\right)^{\vee} \longrightarrow H^{0}\left(\mathcal{N}_{l \backslash \mathbb{P}^{4}}\right)^{\vee} \longrightarrow H^{0}\left(\mathcal{N}_{l \backslash X_{t}}\right)^{\vee} \longrightarrow 0
$$

We may think of $\Omega_{\mathcal{H} / \mathbb{C},\{l\}}^{1}$ as the cokernel of the map $\psi_{t}^{\vee}$, where $\psi_{t}$ is

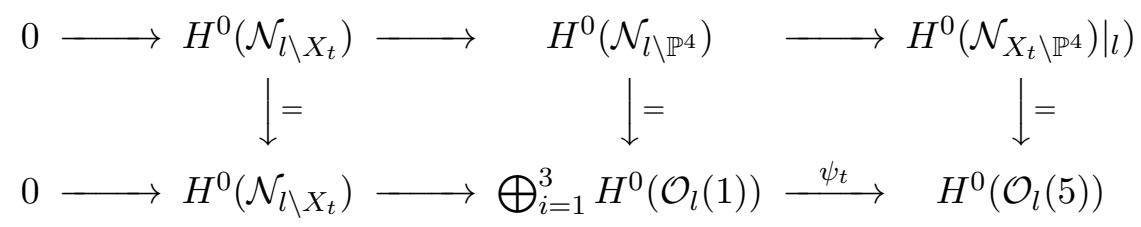

Consider $U_{01}=G(2,5) \backslash\left\{p_{01}=0\right\}$ and $\{l\} \in U_{01}$ generic given by $\phi: \mathbb{P}^{1} \rightarrow \mathbb{P}^{4}$

$$
\phi((\alpha: \beta)):=\left(\alpha: \beta: x_{2} \alpha+y_{2} \beta: x_{3} \alpha+y_{3} \beta: x_{4} \alpha+y_{4} \beta\right)
$$


the line passing through the points $x=\left(1: 0: x_{2}: x_{3}: x_{4}\right)$ and $y=\left(0: 1: y_{2}: y_{3}: y_{4}\right)$, where $\left(x_{2}, x_{3}, x_{4}, y_{2}, y_{3}, y_{4}\right)$ are coordinates for $U_{01}$. Then

$$
\bigoplus_{i=2}^{4} H^{0}\left(\mathcal{O}_{l}(1)\right) \stackrel{\psi_{t}}{\longrightarrow} H^{0}\left(\mathcal{O}_{l}(5)\right)
$$

is

$$
\psi_{t}=\left(\phi^{*}\left(\frac{\partial F_{t}}{\partial z_{2}}\right), \phi^{*}\left(\frac{\partial F_{t}}{\partial z_{3}}\right), \phi^{*}\left(\frac{\partial F_{t}}{\partial z_{4}}\right)\right) .
$$

In particular, if we consider the canonical bases $(\alpha, \beta)$ for $H^{0}\left(\mathcal{O}_{l}(1)\right)$ and $\left(\alpha^{5}, \alpha^{4} \beta, \ldots, \alpha \beta^{4}, \beta^{5}\right)$ for $H^{0}\left(\mathcal{O}_{l}(5)\right)$, then

$$
\bigoplus_{i=2}^{4} H^{0}\left(\mathcal{O}_{l_{2}}(1)\right) \stackrel{\psi_{2 t}}{\longrightarrow} H^{0}\left(\mathcal{O}_{l_{2}}(5)\right),
$$

corresponding to the line $l_{2}$ embedded by $\phi_{2}$, is

$$
\begin{gathered}
\psi_{2 t}\left(\left(a_{2}, b_{2}\right),\left(a_{3}, b_{3}\right),\left(a_{4}, b_{4}\right)\right)=\sum_{i=2}^{4}\left(a_{i} \alpha+b_{i} \beta\right)\left(\phi_{2}^{*}\left(\frac{\partial F_{t}}{\partial z_{i}}\right)\right)= \\
=5\left(a_{2} \alpha+b_{2} \beta\right)\left(\alpha^{4}-t a b \alpha \beta^{3}\right)+ \\
+5\left(a_{3} \alpha+b_{3} \beta\right)\left(a^{4} \alpha^{4}+t b \alpha^{2} \beta^{2}\right)+5\left(a_{4} \alpha+b_{4} \beta\right)\left(b^{4} \beta^{4}+t a \alpha^{2} \beta^{2}\right) ;
\end{gathered}
$$

or, in matrix form with respect to the standard bases of $H^{0}\left(\mathcal{O}_{l_{2}}(1)\right)$ and $H^{0}\left(\mathcal{O}_{l_{2}}(5)\right)$ :

$$
\psi_{2 t}=5\left(\begin{array}{cccccc}
1 & 0 & 0 & -t a b & 0 & 0 \\
0 & 1 & 0 & 0 & -t a b & 0 \\
0 & 0 & t b & 0 & a^{4} & 0 \\
0 & 0 & 0 & t b & 0 & a^{4} \\
0 & 0 & t a & 0 & b^{4} & 0 \\
0 & 0 & 0 & t a & 0 & b^{4}
\end{array}\right)
$$

The determinant of this matrix is $t^{2}\left(a^{5}+b^{5}\right)^{2}=t^{2}$.

$\psi_{1 t}$ may be obtained from the above by setting $a=-1, b=0$. Thus:

$$
\begin{aligned}
& h^{0}\left(\mathcal{N}_{l_{1} \backslash X_{0}}\right)=\operatorname{dim} \operatorname{Ker} \psi_{10}=2, \\
& h^{0}\left(\mathcal{N}_{l_{1} \backslash X_{t}}\right)=\operatorname{dim} \operatorname{Ker} \psi_{1 t}=0,
\end{aligned}
$$

for $t \neq 0$. Furthermore

$$
h^{0}\left(\mathcal{N}_{l_{2} \backslash X_{0}}\right)=\operatorname{dim} \operatorname{Ker} \psi_{20}=2 .
$$

Similarly, $h^{0}\left(\mathcal{N}_{l_{1} \backslash \mathcal{X}}\right), h^{0}\left(\mathcal{N}_{l_{2} \backslash \mathcal{X}}\right)$, can be obtained from the exact sequence

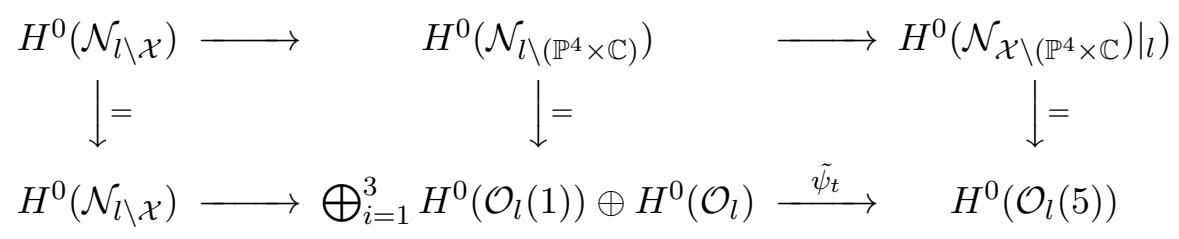


where $\tilde{\psi}_{t}$ is obtained from $\psi_{t}$ by adding the row:

$$
\left(\begin{array}{llllll}
0 & 0 & 0 & 0 & 0 & 0
\end{array}\right)
$$

given by $\phi_{1}^{*}\left(\frac{\partial F_{t}}{\partial t}\right)$ for $l_{1}$, and

$$
\left(\begin{array}{llllll}
0 & 0 & 0 & -a b & 0 & 0
\end{array}\right)
$$

given by $\phi_{2}^{*}\left(\frac{\partial F_{t}}{\partial t}\right)$ for $l_{2}$. Thus:

$$
\begin{aligned}
& h^{0}\left(\mathcal{N}_{l_{1} \backslash \mathcal{X}}\right)=\operatorname{dim} \operatorname{Ker} \tilde{\psi_{1 t}}=3 \\
& h^{0}\left(\mathcal{N}_{l_{2} \backslash \mathcal{X}}\right)=\operatorname{dim} \operatorname{Ker} \tilde{\psi_{2 t}}=2 .
\end{aligned}
$$

Note that A.Albano and S.Katz have shown in AK1 that the local dimension of $\mathcal{H}$ is 2 at each point of $\mathcal{H}_{0}$.

We can summarize the above results in the following:

Lemma 1.2. With the above notations, we have:

$$
\begin{array}{ll}
\mathcal{N}_{l_{1} \backslash X_{0}}=\mathcal{O}_{\mathbb{P}^{1}}(1) \oplus \mathcal{O}_{\mathbb{P}^{1}}(-3), & \mathcal{N}_{l_{1} \backslash \mathcal{X}}=\mathcal{O}_{\mathbb{P}^{1}}(1) \oplus \mathcal{O}_{\mathbb{P}^{1}}(-3) \oplus \mathcal{O}_{\mathbb{P}^{1}} \\
\mathcal{N}_{l_{2} \backslash X_{0}}=\mathcal{O}_{\mathbb{P}^{1}}(1) \oplus \mathcal{O}_{\mathbb{P}^{1}}(-3), & \mathcal{N}_{l_{2} \backslash \mathcal{X}}=\mathcal{O}_{\mathbb{P}^{1}} \oplus \mathcal{O}_{\mathbb{P}^{1}} \oplus \mathcal{O}_{\mathbb{P}^{1}}(-2)
\end{array}
$$

and $\mathcal{N}_{l_{1} \backslash X_{t}}=\mathcal{O}_{\mathbb{P}^{1}}(-1) \oplus \mathcal{O}_{\mathbb{P}^{1}}(-1)$ for $t \neq 0$.

Thus the relative Hilbert scheme $\mathcal{H}$ of the Dwork pencil is smooth at the points of $\mathcal{H}_{0}$ other than its crossing points. It contains as irreducible components 375 smooth curves corresponding to the base locus of the family $\mathcal{H}_{t}$. These curves intersect the rest of $\mathcal{H}$ only in the 375 crossing points of $\mathcal{H}_{0}$.

Proof. By Grothendieck's lemma,

$$
\mathcal{N}_{l \backslash X_{t}}=\mathcal{O}_{\mathbb{P}^{1}}(a) \oplus \mathcal{O}_{\mathbb{P}^{1}}(b)
$$

for any of the above mentioned lines $l \subset X_{t}$, where $a+b=-2$ by adjunction. This, together with formulas (1.2)-(1.6) are enough to establish the form of $\mathcal{N}_{l \backslash X_{t}}$. For $\mathcal{N}_{l \backslash \mathcal{X}}$, use that

$$
\mathcal{N}_{l \backslash \mathcal{X}}=\mathcal{O}_{\mathbb{P}^{1}}(c) \oplus \mathcal{O}_{\mathbb{P}^{1}}(d) \oplus \mathcal{O}_{\mathbb{P}^{1}}(e)
$$

with $a \leq c, b \leq d$ and $c+d+e=-2$, since $\mathcal{N}_{l \backslash \mathcal{X}}$ is the middle term in the exact sequence

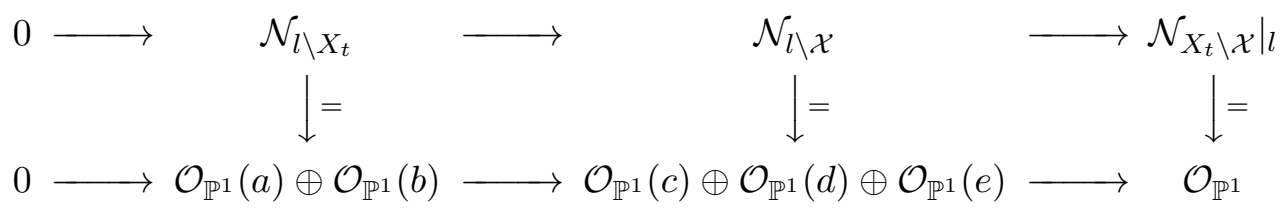

The local proprieties of $\mathcal{H}$ are deduced via the identifications of the tangent spaces: $T_{\mathcal{H}_{t},\{l\}}=H^{0}\left(\mathcal{N}_{l \backslash X_{t}}\right), T_{\mathcal{H},\{l\}}=H^{0}\left(\mathcal{N}_{l \backslash \mathcal{X}}\right)$.

In fact, some of the above results have already been obtained via different methods in AK2. 
2. The Relative Hilbert scheme $\mathcal{H}$ in a neighborhood of $\mathcal{H}_{0}$ : the HiLBERT SCHEME AS A GRADIENT SCHEME

In $\mathrm{C} 2$, the relative Hilbert scheme of curves on a family of Calabi-Yau threefolds is constructed locally analytically as a (relative) gradient scheme, more precisely as the zero scheme of the exterior derivative of an analytic function on an analytic family of differentiable curves in the threefolds. In this section we will see how the description of $\mathcal{H}$ locally as a gradient scheme can give us information on its behavior in the neighborhood of $\mathcal{H}_{0}$.

The following lemma will prove useful in this context:

Lemma 2.1. Let $\mathcal{C}$ denote one of the 375 curves identified above as components of the relative Hilbert scheme. Let $\mathcal{M} \subset \mathcal{O}_{\mathcal{C}}$ denote the maximal ideal at $t=0$. Then in a neighborhood of 0 , the maximal ideal $\mathcal{M}$ annihilates the stalk of $\left(\Omega_{\mathcal{H} / \mathbb{P}^{1}}^{1} \otimes \mathcal{O}_{\mathcal{C}}\right)$.

Proof. With the same notations as in the preceding section,

$$
H^{0}\left(U_{01} \cap \mathcal{C}, \Omega_{\mathcal{H} / \mathbb{C}}^{1} \otimes \mathcal{O}_{U_{01} \cap \mathcal{C}}\right)=\frac{\mathbb{C}<d x_{2}, d y_{2}, d x_{3}, d y_{3}, d x_{4}, d y_{4}>}{\operatorname{Im}\left(\psi_{2 t}\right)^{*}}
$$

On $\mathcal{C}, \psi_{2 t}$ is given (with respect to the canonical bases) by

$$
\psi_{2 t}=5\left(\begin{array}{cccccc}
1 & 0 & 0 & 0 & 0 & 0 \\
0 & 1 & 0 & 0 & 0 & 0 \\
0 & 0 & 0 & 0 & 1 & 0 \\
0 & 0 & 0 & 0 & 0 & 1 \\
0 & 0 & -t & 0 & 0 & 0 \\
0 & 0 & 0 & -t & 0 & 0
\end{array}\right)
$$

Then by Lemma 1.1

$$
H^{0}\left(U_{01} \cap \mathcal{C}, \Omega_{\mathcal{H} / \mathbb{C}}^{1} \otimes \mathcal{O}_{U_{01} \cap \mathcal{C}}\right) \cong \frac{\mathbb{C}[t]<d x_{4}, d y_{4}>}{<t d x_{4}, t d y_{4}>}
$$

and therefore $\mathcal{M}$ annihilates the sections of $\left(\Omega_{\mathcal{H} / \mathbb{C}}^{1} \otimes \mathcal{O}_{U_{01} \cap \mathcal{C}}\right)$.

The preceding lemma and the fact that locally analytically the Hilbert scheme may be written as a gradient scheme can be used to establish the following proposition:

Proposition 2.2. Let $l_{1}$ be one of the crossing points of $\mathcal{H}_{0}$. Then there exists an analytical neighborhood $U$ of $l_{1}$ such that for $t$ close to $0, \mathcal{H}_{t} \cap U$ consists of two smooth disjoint curves and an isolated zero, (which is $\mathcal{C} \cap \mathcal{H}_{t}$ ). Moreover, $(\mathcal{H} \backslash \mathcal{C}) \cap U$ is a smooth irreducible surface.

Proof. As mentioned before, in an analytical neighborhood of $l_{1}$, the Hilbert scheme $\mathcal{H}_{0}$ can be described as Spec $\mathbb{C}[[x, y]] /\left(x^{3} y^{2}, x^{2} y^{3}\right)$ (where the coordinates $x, y$ can be thought of as coordinates in an analytic disc of dimension $\left.2=h^{0}\left(\mathcal{N}_{l_{1} \backslash X_{0}}\right)\right)$. 
Following [C2, an easy exercise shows that a potential function $\Phi^{0}$, whose gradient gives $\mathcal{H}_{0}$, can be written, after an appropriate choice of coordinates, as $x^{3} y^{3}$. Furthermore, one can extend the function $x^{3} y^{3}$ to some analytic function $\Phi(x, y, t)$ such that

$$
\Phi(x, y, t)=x^{3} y^{3}+\operatorname{tg}(x, y)+t^{2} h(x, y, t)
$$

and, in some analytical neighborhood $U$ of $l_{1}, \mathcal{H}$ is given by the zeroes of the differential of $\Phi$ in the direction of the fiber:

$$
\left(\Phi_{x}=\Phi_{y}=0\right) .
$$

Moreover, we can choose coordinates $x, y$, such that $\mathcal{C}$ is given by $(x=y=0)$ on $\mathrm{U}$. This means that $g_{x}(0,0)=g_{y}(0,0)=0$, i.e.

$$
g(x, y)=a x^{2}+2 b x y+c y^{2}+o(3) .
$$

In this setting, $\Omega_{\mathcal{H} / \mathbb{C}}^{1} \otimes \mathcal{O}_{\mathcal{C}}$ over $\mathrm{U}$ has the form

$$
\frac{\mathbb{C}[t]<d x, d y>}{<\begin{array}{l}
\left(t g_{x x}(0,0)+t^{2} h_{x x}(0,0, t)\right) d x+\left(t g_{x y}(0,0)+t^{2} h_{x y}(0,0, t)\right) d y \\
\left(t g_{x y}(0,0)+t^{2} h_{x y}(0,0, t)\right) d x+\left(t g_{y y}(0,0)+t^{2} h_{y y}(0,0, t)\right) d y
\end{array}>}
$$

and under these conditions Lemma 2.1. implies that

$$
\left|\begin{array}{ll}
g_{x x}(0,0) & g_{x y}(0,0) \\
g_{x y}(0,0) & g_{y y}(0,0)
\end{array}\right|=a c-b^{2} \neq 0
$$

otherwise t would be a nilpotent element of $\Omega_{\mathcal{H} / \mathbb{P}^{1}}^{1} \otimes \mathcal{O}_{\mathcal{C}}$ over $\mathrm{U}$.

Then

$$
\begin{aligned}
& \Phi_{x}=3 x^{2} y^{3}+2 t(a x+b y+o(2))+t^{2} h_{x}(x, y, t), \\
& \Phi_{y}=3 x^{3} y^{2}+2 t(b x+c y+o(2))+t^{2} h_{y}(x, y, t) .
\end{aligned}
$$

In other words,

$$
\begin{aligned}
& \Phi_{x}=3 x^{2} y^{3}+t(A x+B y), \\
& \Phi_{y}=3 x^{3} y^{2}+t(C x+D y),
\end{aligned}
$$

where $A, B, C, D$ are analytical functions in $x, y, t$, with $A D-B C$ invertible in an analytic neighborhood of $(0,0,0)$.

Notice

$$
C \Phi_{x}-A \Phi_{y}=3 x^{2} y^{2}(C y-A x)+t(C B-A D) y=y k_{1}(x, y, t),
$$

where $k_{1}(x, y, t):=3 x^{2} y(C y-A x)+t(C B-A D)$.

$$
D \Phi_{x}-B \Phi_{y}=3 x^{2} y^{2}(D y-B x)-t(C B-A D) x=x k_{2}(x, y, t),
$$

where $k_{2}(x, y, t):=3 x y^{2}(D y-B x)-t(C B-A D)$.

Since $A D-B C$ Ás invertible in an analytic neighborhood of $(0,0,0)$, it follows that the Hilbert scheme $\mathcal{H}_{t}$ is given in that neighborhood by the ideal

$$
\left(y k_{1}(x, y, t), x k_{2}(x, y, t)\right)
$$


On the other hand, $\mathcal{H}$ has at least one local component of dimension 2 , that cannot be given by $x=0$ or $y=0$ since $x=y=0$ is isolated in $\mathcal{H}_{t}$ for small non-zero $t$. So

$$
k_{1}=E k_{2}
$$

for a unit $E$, and the zeroes of $k_{1}$ give a smooth surface with tangent plane $(t=0)$ at $(0,0,0)$. By inspection on $k_{1}$ and $k_{2}$ we get that $E$ must equal 1, $A=D=0$ and $B=C$.

Thus for $U$ small enough, $\mathcal{H} \cap U$ has irreducible components given by $(x=y=0)$ and $\left(k_{1}=0\right)$, and $\mathcal{H}_{t} \cap U$ consists the two smooth disjoint curves

$$
x y= \pm t^{1 / 2}
$$

together with the isolated point $x=y=0$.

Proposition 2.3. For $t \neq 0$ in an analytical neighborhood of 0 , the relative Hilbert scheme $\mathcal{H}_{t}$ is a smooth curve, together with 375 isolated points.

Proof. It remains to prove that as it departs from a point $l_{2}$ in the $t$-direction, the non-reduced curve $\mathcal{H}_{0}$ splits locally analytically into two non-intersecting analytical open subsets of $\mathcal{H}_{t}$, both of which are smooth, 1-dimensional.

In an analytical neighborhood of $l_{2}$, the Hilbert scheme $\mathcal{H}_{0}$ can be described as Spec $\mathbb{C}[[x, y]] /\left(y^{2}\right)$. Here again, as in the previous proposition, the coordinates $x, y$ can be thought of as coordinates in an analytic disc of dimension $2=h^{0}\left(\mathcal{N}_{l_{2} \backslash X_{0}}\right)$, which is in accord with the data in [C2]. Thus one can again extend the function $x^{3}$ to

$$
\Phi(x, y, t)=x^{3}+t g(x, y)+t^{2} h(x, y, t)
$$

analytical such that, in an analytical neighborhood $U$ of $l_{2}, \mathcal{H}$ is given by $\left(\Phi_{x}=\Phi_{y}=0\right)$, with

$$
\begin{gathered}
\Phi_{x}(x, y, t)=3 x^{2}+t g_{x}(x, y)+t^{2} h_{x}(x, y), \\
\Phi_{y}(x, y, t)=t g_{y}(x, y)+t^{2} h_{y}(x, y) .
\end{gathered}
$$

For any $\{l\} \in U \cap \mathcal{H}, \operatorname{dim} T_{\mathcal{H}, l}=3-r(x, y, t)$ where $r(x, y, t)$ is the rank of the following matrix

$$
\left(\begin{array}{ll}
6 x+t g_{x x}+t^{2} h_{x x} & t g_{x y}+t^{2} h_{x y} \\
t g_{x y}+t^{2} h_{x y} & t g_{y y}+t^{2} h_{y y} \\
g_{x}+2 t h_{x}+t^{2} h_{t x} & g_{y}+2 t h_{y}+t^{2} h_{t y}
\end{array}\right) .
$$

Since $(0,0,0)$ is a smooth point of $\mathcal{H}$, we necessarily have:

$$
r(0,0,0)=\operatorname{rank}\left(g_{x}(0,0) \quad g_{y}(0,0)\right)=1 .
$$

On the other hand, $(x, y, 0)$ is also a smooth point of $\mathcal{H}$ for any $x, y$ small enough, so

$$
r(x, y, 0)=\operatorname{rank}\left(\begin{array}{cc}
6 x & 0 \\
0 & 0 \\
g_{x}(x, y) & g_{y}(x, y)
\end{array}\right)=1 .
$$


Then $g_{y}(x, y)=0$ for $x \neq 0$ and since $g$ is analytic in $x$ and $y$, this shows that $g$ depends only on the variable $x$ and that $g_{x}(0) \neq 0$. Thus

$$
\Phi_{x}(x, y, t)=3 x^{2}+t g_{x}(x)+t^{2} h_{x}(x, y, t)=3 x^{2}+t A(x, y, t),
$$

where $A(x, y, t)$ is analytic and invertible in a neighborhood of $(0,0,0)$.

$$
\Phi_{y}(x, y, t)=t^{2} h_{y}(x, y, t) .
$$

After an analytic change of variables we can rewrite the local equations of $\mathcal{H}$ on $U$ as $\left(x^{\prime 2}-t=0, t^{2} f\left(x^{\prime}, y, t\right)=0\right)$. As this should be a surface around $(x, y, 0), f \in\left(x^{2}-t\right)$ and this concludes the proof.

By combining the local information on the relative Hilbert scheme $\mathcal{H}$ and that on the associated normal function derived from the above propositions, we obtain the following

Corollary 2.4. The general fiber $\mathcal{H}_{t}$ of the family $\mathcal{H}$ is reducible.

Proof. As before, let $l_{1}$ denote a point on $\mathcal{H}_{0}$ which is one of the 375 basepoints of $\mathcal{H}$ and let $l_{2}$ denote a general point of $\mathcal{H}_{0}$. We define the analytic function

$$
\begin{gathered}
F: \mathcal{H} \rightarrow \mathbb{C} \\
(l, t) \rightarrow \int_{\left(l_{1}, t\right)}^{(l, t)} \omega(t)
\end{gathered}
$$

where $\omega(t)$ is a holomorphic 3 -form on $X_{t}$, varying holomorphically with $t$. From the previous proposition we know that, in appropriate analytic coordinates $(x, y, t), \mathrm{F}$ is given in a neighborhood of $\left(l_{2}, 0\right)$ by

$$
x^{3}-\left.t x\right|_{3 x^{2}-t=0} .
$$

Therefore

$$
F(x, y, t)=x^{3}-3 x^{2} x=-2 x^{3}
$$

is not constant in a neighborhood of $\left(l_{2}, 0\right)$, because of the branching given by

$$
x=\left(\frac{t}{3}\right)^{1 / 2} .
$$

Since $F$ should be constant on each component of $\mathcal{H}_{t}$, it follows that $\mathcal{H}_{t}$ has at least two different connected components.

A more detailed algebraic analysis of the Stein factorization of $\mathcal{H} \rightarrow \mathbb{C}$ will be given in sections 6 and 7 . 


\section{The VAN GEEMEN LINES}

In AK2 it is proven that the generic Hilbert scheme $\mathcal{H}_{t}$ has a component of dimension 1. The proof is based on identifying a particular set of 5000 points on each $\mathcal{H}_{t}$-the Van Geemen lines- and proving that, for $t$ generic, the dimension of the tangent space to $\mathcal{H}_{t}$ at those points is 1 . We know that the generic quintic threefold contains 2875 lines. Since no smooth quintic admits a 2-parameter family of lines, this shows that $\mathcal{H}_{t}$ has a component of dimension 1.

Following is a brief description of how the van Geemen lines occur in the quintic $X_{t}$. After that, $\mathcal{N}_{l_{3} \backslash X_{t}}$ and $\mathcal{N}_{l_{3} \backslash \mathcal{X}}$ are computed by the methods introduced in section 1 , to the effect that all van Geemen lines are smooth points of $\mathcal{H}$. Also, all but those over the fibers at the fifth roots of $\frac{2^{7}}{3}$ are smooth points of their fibers $\mathcal{H}_{t}$.

Consider lines passing through points of the form: $(1: 1: 1: a: b)$ and $\left(1: \xi: \xi^{2}: 0: 0\right)$ in $\mathbb{P}^{4}$, where $\xi$ is a complex number satisfying $1+\xi+\xi^{2}=0$. The conditions for such a line to be contained in one of the $X_{t}$-s are:

$$
b^{5}+a^{5}=27 \text { and } t b a=6 .
$$

Let $l_{3}$ denote such a line. There are 10 distinct solutions of the above equation for each $t \neq 0$. Using the action of the group $G \times S_{5}$ one obtains a set of 5,000 lines in each $X_{t}$ (see AK2). These will be called the van Geemen lines.

Consider the Plücker embedding of the Grassmannian $G(2,5)$ in $\mathbb{P}^{9}$. Notice that all the van Geemen lines correspond to points inside the 10-degree hypersurface in $\mathbb{P}^{9}$ :

$$
P=\bigcup_{i, j=0}^{4}\left\{p_{i j}=0\right\}
$$

On the other hand, we know that $\mathcal{H}_{0} \subset G(2,5)$ consists of 50 quintic curves with multiplicity 2 , thus the intersection cycle with $\mathrm{P}$ has number 5000 . We will see shortly that, indeed, the van Geemen points in $\mathcal{H}_{t}$ give exactly the above mentioned hyperplane sections on $\mathcal{H}_{t}$.

We next compute the normal bundles to lines $l_{3}$ in $X_{t}, \mathcal{X}$, using Lemma 1.1 .

Let $l_{3}$ be given by the morphism $\phi_{3}: \mathbb{P}^{1} \rightarrow \mathbb{P}^{4}$,

$$
\phi_{3}((\alpha: \beta)):=\left(\alpha+\beta \xi^{2}: \alpha+\beta \xi: \alpha+\beta: \alpha a: \alpha b\right),
$$

with $b^{5}+a^{5}=27, t b a=6$.

As before, one works with the exact sequence:

$$
0 \longrightarrow H^{0}\left(\mathcal{N}_{l_{3} \backslash X_{t}}\right) \longrightarrow \bigoplus_{i=1}^{3} H^{0}\left(\mathcal{O}_{l_{3}}(1)\right) \stackrel{\psi_{3 t}}{\longrightarrow} H^{0}\left(\mathcal{O}_{l_{3}}(5)\right) \text {. }
$$

Then as before

$$
\psi_{3 t}\left(\left(a_{2}, b_{2}\right),\left(a_{3}, b_{3}\right),\left(a_{4}, b_{4}\right)\right)=\sum_{i=2}^{4}\left(a_{i} u+b_{i} v\right)\left(\phi_{3}^{*}\left(\frac{\partial F_{t}}{\partial z_{i}}\right)\right)=
$$




$$
\begin{gathered}
=5\left(a_{2} u+b_{2} v\right)\left(-5 u^{4}+10 u^{3} v+4 u v^{3}+v^{4}\right)+ \\
+5\left(a_{3} u+b_{3} v\right)\left(\left(a^{4}-t b\right) u^{4}-t b u v^{3}\right)+5\left(a_{4} u+b_{4} v\right)\left(\left(b^{4}-t a\right) u^{4}-t a u v^{3}\right) .
\end{gathered}
$$

In terms of the basis $(\alpha, \beta)$ for $H^{0}\left(\mathcal{O}_{l_{3}}(1)\right)$ and $\left(\alpha^{5}, \alpha^{4} \beta, \ldots, \alpha \beta^{4}, \beta^{5}\right)$ for $H^{0}\left(\mathcal{O}_{l_{3}}(5)\right)$ :

$$
\psi_{3 t}=5\left(\begin{array}{cccccc}
-5 & 10 & 0 & 4 & 1 & 0 \\
0 & -5 & 10 & 0 & 4 & 1 \\
a^{4}-t b & 0 & 0 & -t b & 0 & 0 \\
0 & a^{4}-t b & 0 & 0 & -t b & 0 \\
b^{4}-t a & 0 & 0 & -t a & 0 & 0 \\
0 & b^{4}-t a & 0 & 0 & -t a & 0
\end{array}\right) .
$$

Notice that since the 3 -rd and the 6 -th columns of the above matrix are linearly dependent,

$$
h^{0}\left(\mathcal{N}_{l_{3} \backslash X_{t}}\right)=\operatorname{dim} \operatorname{Ker} \psi_{3 t} \geq 1
$$

for all $t \neq 0$. Also notice that if

$$
a^{5}=b^{5}
$$

then rows 3 and 5 are linearly dependent and the same is true of rows 4 and 6 . In this case one can immediately check that the rank of the above matrix is 4 and so

$$
h^{0}\left(\mathcal{N}_{l_{3} \backslash X_{t}}\right)=\operatorname{dim} \operatorname{Ker} \psi_{3 t}=2 .
$$

Under the condition $a^{5}=b^{5}$, the equations for the van Geemen lines:

$$
b^{5}+a^{5}=27, t b a=6
$$

yield solutions

and

$$
a=\mu_{1} \sqrt[5]{\frac{27}{2}}, b=\mu_{2} \sqrt[5]{\frac{27}{2}}
$$

$$
t=\sqrt[5]{\frac{2^{7}}{3}} \mu
$$

where $\mu_{1} \mu_{2} \mu=1$ and $\mu_{1}, \mu_{2}$ are 5 -th roots of unity.

Otherwise, we check that the determinant

$$
\begin{gathered}
\left|\begin{array}{ccccc}
0 & -5 & 0 & 4 & 1 \\
a^{4}-t b & 0 & -t b & 0 & 0 \\
0 & a^{4}-t b & 0 & -t b & 0 \\
b^{4}-t a & 0 & -t a & 0 & 0 \\
0 & b^{4}-t a & 0 & -t a & 0
\end{array}\right|=\left|\begin{array}{ccccc}
0 & -9 & 0 & 4 & 1 \\
a^{4} & 0 & -t b & 0 & 0 \\
0 & a^{4} & 0 & -t b & 0 \\
b^{4} & 0 & -t a & 0 & 0 \\
0 & b^{4} & 0 & -t a & 0
\end{array}\right|= \\
=t^{2}\left(a^{10}-b^{10}\right)=27 t^{2}\left(a^{5}-b^{5}\right) \neq 0 .
\end{gathered}
$$

Thus for $t \neq \sqrt[5]{\frac{2^{7}}{3}} \mu$,

$$
h^{0}\left(\mathcal{N}_{l_{3} \backslash X_{t}}\right)=\operatorname{dim} \operatorname{Ker} \psi_{3 t}=1 .
$$


Similarly, $h^{0}\left(\mathcal{N}_{l_{3} \backslash \mathcal{X}}\right)$ can be obtained from the exact sequence

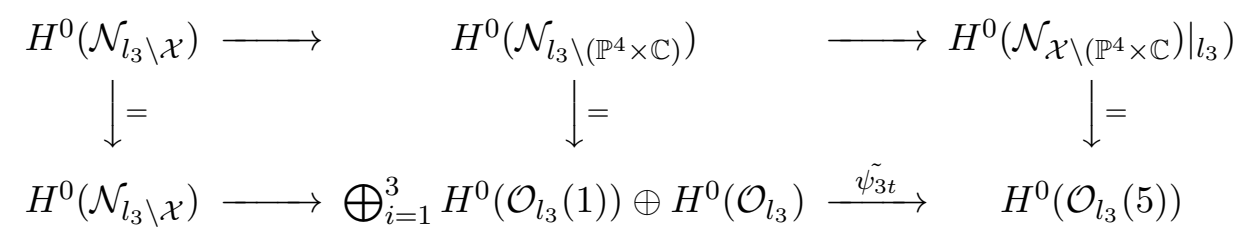

where the left horizontal morphisms are injective and $\tilde{\psi_{3 t}}$ is obtained from $\psi_{3 t}$ by adding the row:

$$
\left(\begin{array}{llllll}
-a b & 0 & 0 & -a b & 0 & 0
\end{array}\right)
$$

given by $\phi_{3}^{*}\left(\frac{\partial F_{t}}{\partial t}\right)$, so:

$$
h^{0}\left(\mathcal{N}_{l_{3} \backslash \mathcal{X}}\right)=\operatorname{dim} \operatorname{Ker} \tilde{\psi_{3 t}}=2 .
$$

These results can be summarized in the following:

Lemma 3.1. With the above notations, we have:

$$
\begin{aligned}
\mathcal{N}_{l_{3} \backslash X_{t}} & =\mathcal{O}_{\mathbb{P}^{1}}(1) \oplus \mathcal{O}_{\mathbb{P}^{1}}(-3) \text { for } t=2 \sqrt[5]{\frac{4}{3}} \mu, \\
\mathcal{N}_{l_{3} \backslash X_{t}} & =\mathcal{O}_{\mathbb{P}^{1}} \oplus \mathcal{O}_{\mathbb{P}^{1}}(-2) \text { for } t \neq 2 \sqrt[5]{\frac{4}{3}} \mu, \\
\mathcal{N}_{l_{3} \backslash \mathcal{X}} & =\mathcal{O}_{\mathbb{P}^{1}} \oplus \mathcal{O}_{\mathbb{P}^{1}} \oplus \mathcal{O}_{\mathbb{P}^{1}}(-2) \text { for all } t .
\end{aligned}
$$

Thus the relative Hilbert scheme $\mathcal{H}$ of the Dwork pencil is a smooth surface at the Van Geemen points; all the fibers $\mathcal{H}_{t}$ are smooth at these points except those where $t$ is a fifth root of $\frac{2^{7}}{3}$.

\section{Algebraic description of the universal line $\mathcal{U}$ away from $\mathcal{U}_{0}$ AND THE VAN GEEMEN LINES}

Notation . As before, let $G(2,5)$ denote the Grassmannian of lines in $\mathbb{P}^{4}$. Let $F(1,2,5) \subset G(2,5) \times \mathbb{P}^{4}$ denote the flag variety

$$
F(1,2,5)=\{(\{l\}, y) /\{l\} \in G(2,5), y \in l .\}
$$

Let $\mathcal{U} \subset \mathcal{H} \times \mathbb{P}^{4}$ denote the universal line of $\mathcal{H}$. Let $\overline{\mathcal{U}} \subset \overline{\mathcal{H}} \times \mathbb{P}^{4}$ denote the closure of $\mathcal{U}$ in $F(1,2,5)$.

Let $U$ be the projection of $\mathcal{U} \subset F(1,2,5) \times \mathbb{P}^{1}$ to $F(1,2,5)$.

Notation . For each $i \in\{0, \ldots, 4\}$, let $\Psi^{i}$ denote the rational map from $F(1,2,5)$ to $\mathbb{P}^{3}$, defined by

$$
\Psi^{i}((\{l\}, y))=\left(\frac{x_{0}}{y_{0}}: \ldots: \frac{\hat{x}_{i}}{y_{i}}: \ldots: \frac{x_{4}}{y_{4}}\right),
$$

where $y=\left(y_{0}: \ldots: y_{4}\right) \in l \subset \mathbb{P}^{4}$ and $x=\left(x_{0}: \ldots: x_{4}\right) \in l \cap H_{i}$. 
The restriction on $U$ of the rational map $\Psi^{i}$ will be the main tool in our algebraic study of $\mathcal{U}$ away from the Van Geemen lines and from the lines in $X_{0}$. We will denote this restriction by $\psi^{i}$.

We can understand the map $\Psi^{i}$ geometrically as follows: for a given line $l \subset \mathbb{P}^{4} \backslash\left(\bigcup_{j=0}^{4} H_{j}\right)$ and a fixed point $y \in l$, we consider $y$ to be the point at $\infty$ on $l$, and $l \cap H_{i}$ to be the 0 point on $l$. Modulo the action of $\mathbb{C}^{*}$, this gives a parametrization on $l$. Thus, 4-tuples of points on $l$, none of which is $\infty$, will correspond via this structure on $l$ to points of $\mathbb{P}^{3}$. We let $\Psi^{i}((\{l\}, y)) \in \mathbb{P}^{3}$ be given by the 4 -tuple of intersection points $\left\{l \cap H_{j}\right\}$, where $j \neq i$. Note that this construction only works for $l \not \subset \bigcup_{j=0}^{4} H_{j}$ and $y \in \mathbb{P}^{4} \backslash\left(\bigcup_{j=0}^{4} H_{j}\right)$. It extends algebraically to $(\{l\}, y)$ with $y \in H_{j} \backslash\left(\bigcup_{k \neq j} H_{k}\right)$, as long as $l \not \subset \bigcup_{j \neq i} H_{j}$.

We may think of $F(1,2,5)$ as the projectivization of the tangent bundle $T_{\mathbb{P}^{4}}$. Over

$$
\left(\mathbb{C}^{*}\right)^{4}=\mathbb{P}^{4} \backslash\left(\bigcup_{j=0}^{4} H_{j}\right),
$$

this bundle is trivial and any of the maps $\Psi^{i}$ gives a trivialization of it.

Notation . Let $\mathcal{U}^{\prime} \subset \mathcal{U}$ be defined as:

$$
\mathcal{U}^{\prime}=\left\{(\{l\}, y) \in \mathcal{U} / \prod_{j=0}^{4} y_{j} \neq 0\right\} .
$$

Let $U^{\prime} \subset U$ be the projection of $\mathcal{U}^{\prime}$ on $F(1,2,5)$.

Notation . Consider the Plücker embedding of $G(2,5)$ in $\mathbb{P}^{9}$, with coordinates $p_{i j}$. Let $\mathcal{H}^{\prime} \subset \mathcal{H}$ be defined as the preimage in $\mathcal{H}$ of

$$
G(2,5) \backslash\left(\bigcup_{i, j=0}^{4}\left\{p_{i j}=0\right\}\right) .
$$

The following notations will prove very useful in better understanding the subset $\mathcal{U}^{\prime} \subset \mathcal{U}$ and the morphisms

$$
\left.\psi^{i}\right|_{U^{\prime}}: U^{\prime} \rightarrow \mathbb{P}^{3}
$$

Notation . Let $(\{l\}, y) \in F(1,2,5)$ be such that $\prod_{j=0}^{4} y_{j} \neq 0$. For each $x=\left(x_{0}: \ldots: x_{4}\right) \in l$, let

$$
u_{j}=\frac{x_{j}}{y_{j}}, \forall j \in\{0, \ldots, 4\} .
$$

Following the discussion above, if $x$ varies in one of the coordinate hyperplanes $H_{i} \subset \mathbb{P}^{4}$, then $\left(\left(u_{0}: \ldots: \hat{u}_{i}: \ldots: u_{4}\right),\left(y_{0}: \ldots: y_{4}\right)\right) \in \mathbb{P}^{3} \times \mathbb{P}^{4}$ give coordinates for the restriction of the $\mathbb{P}^{3}$-bundle $F(1,2,5)$ over $\mathbb{P}^{4}$ to $\left(\mathbb{C}^{*}\right)^{4}=\mathbb{P}^{4} \backslash\left(\bigcup_{j=0}^{4} H_{j}\right)$. Notice also that for $x \neq y$ varying in a hyperplane 
$H_{i} \subset \mathbb{P}^{4}$, the projective coordinates $\left(u_{0}: \ldots: \hat{u}_{i}: \ldots: u_{4}\right)$ together with $\left(y_{0}^{5}: \ldots: y_{4}^{5}\right)$ exactly determine the orbit of the line $l$ by the action of the group $\hat{G}$. Let

$$
s_{0}(u)=1, s_{1}(u)=\sum_{i=0}^{4} u_{i}, s_{2}(u)=\sum_{i \neq j ; i, j=0}^{4} u_{i} u_{j}, \text { etc. }
$$

be the fundamental symmetrical polynomials in $\left(u_{0}, \ldots, u_{4}\right)$.

For any $k \in\{0, \ldots 4\}$, let

$$
\sigma_{k}(u, y)=\sum_{i=0}^{4} u_{i}^{k} y_{i}^{5}
$$

For $i, j \in\{0, \ldots 4\}$, we let

$$
u^{i}=\left(u_{0}: \ldots: 0: \ldots: u_{4}\right)
$$

where $u_{i}=0$ and

$$
u^{i j}=\left(u_{0}: \ldots: 0: \ldots: 0: \ldots: u_{4}\right)
$$

where $u_{i}=u_{j}=0$.

Also, let

etc.

$$
\begin{gathered}
\delta(u):=\prod_{j>k}\left(u_{j}-u_{k}\right), \\
\delta\left(u^{i}\right):=\prod_{j, k \neq i ; j>k}\left(u_{j}-u_{k}\right),
\end{gathered}
$$

Notation . If $x \neq y$, we may consider the line $l$ passing through $x$ and $y$ to be parametrized with coordinates $(\alpha: \beta)$ and embedded in $\mathbb{P}^{4}$ by the morphism $\phi: \mathbb{P}^{1} \rightarrow \mathbb{P}^{4}$,

$\phi((\alpha: \beta)):=\left(x_{0} \alpha+y_{0} \beta: x_{1} \alpha+y_{1} \beta: x_{2} \alpha+y_{2} \beta: x_{3} \alpha+y_{3} \beta: x_{4} \alpha+y_{4} \beta\right)$.

In the new coordinates $\left(\left(y_{j}\right)_{j},\left(u_{j}\right)_{j}\right)$ :

$$
\phi((\alpha: \beta))=\left(y_{0}\left(u_{0} \alpha+\beta\right): y_{1}\left(u_{1} \alpha+\beta\right): \ldots: y_{4}\left(u_{4} \alpha+\beta\right)\right) .
$$

Notation . Let $M(u)$ denote the matrix

$$
M(u)=\left(\begin{array}{ccccc}
1 & 1 & 1 & 1 & 1 \\
u_{0} & u_{1} & u_{2} & u_{3} & u_{4} \\
u_{0}^{2} & u_{1}^{2} & u_{2}^{2} & u_{3}^{2} & u_{4}^{2} \\
u_{0}^{3} & u_{1}^{3} & u_{2}^{3} & u_{3}^{3} & u_{4}^{3} \\
u_{0}^{4} & u_{1}^{4} & u_{2}^{4} & u_{3}^{4} & u_{4}^{4}
\end{array}\right) .
$$

Let

$$
Y(y)=\left(\begin{array}{c}
y_{0}^{5} \\
y_{1}^{5} \\
y_{2}^{5} \\
y_{3}^{5} \\
y_{4}^{5}
\end{array}\right) \text { and } C(u)=\left(\begin{array}{c}
s_{0}(u) \\
\frac{1}{5} s_{1}(u) \\
\frac{1}{10} s_{2}(u) \\
\frac{1}{10} s_{3}(u) \\
\frac{1}{5} s_{4}(u)
\end{array}\right)
$$


Proposition 4.1. $\mathcal{U}^{\prime}$ is a 3-dimensional quasi-projective variety, reduced and complete intersection in $F(1,2,5)_{\mid\left(\mathbb{C}^{*}\right)^{4}}$, given, via the trivialization $\Psi_{i}$ of $F(1,2,5)_{\mid\left(\mathbb{C}^{*}\right)^{4}}$, by the following matrix equation on $\mathbb{P}^{3} \times\left(\mathbb{C}^{*}\right)^{4}$ :

$$
M\left(u^{i}\right) \cdot Y(y)=5 t \prod_{j=0}^{4} y_{j} C\left(u^{i}\right) .
$$

Proof. Consider the pair

$$
\left.(\{l\}, y) \in F(1,2,5)\right|_{\left(\mathbb{C}^{*}\right)^{4}},
$$

with the line $l$ parametrized and embedded in $\mathbb{P}^{4}$ by the morphism $\phi: \mathbb{P}^{1} \rightarrow$ $\mathbb{P}^{4}$ given in equation (4.1). The line $l$ is in one of the $X_{t}$-s, for some $t \in \mathbb{C}$, if $\phi^{*}\left(F_{t}\right) \equiv 0$ as a degree 5 homogeneous polynomial in $(\alpha: \beta)$. This gives six equations in $u=\left(u_{0}: \ldots: u_{4}\right), y=\left(y_{0}: . .: y_{4}\right)$ :

$$
\left\{\left(\begin{array}{c}
5 \\
k
\end{array}\right) \sigma_{k}(u, y)-5 t s_{k} \prod_{j=0}^{4} y_{j}=0, \text { for } k \in\{0, . ., 5\} .\right.
$$

Equivalently, in matrix form:

$$
\left(\begin{array}{ccccc}
1 & 1 & 1 & 1 & 1 \\
u_{0} & u_{1} & u_{2} & u_{3} & u_{4} \\
u_{0}^{2} & u_{1}^{2} & u_{2}^{2} & u_{3}^{2} & u_{4}^{2} \\
u_{0}^{3} & u_{1}^{3} & u_{2}^{3} & u_{3}^{3} & u_{4}^{3} \\
u_{0}^{4} & u_{1}^{4} & u_{2}^{4} & u_{3}^{4} & u_{4}^{4} \\
u_{0}^{5} & u_{1}^{5} & u_{2}^{5} & u_{3}^{5} & u_{4}^{5}
\end{array}\right)\left(\begin{array}{c}
y_{0}^{5} \\
y_{1}^{5} \\
y_{2}^{5} \\
y_{3}^{5} \\
y_{4}^{5}
\end{array}\right)=5 t \prod_{j=0}^{4} y_{j}\left(\begin{array}{c}
s_{0}(u) \\
\frac{1}{5} s_{1}(u) \\
\frac{1}{10} s_{2}(u) \\
\frac{1}{10} s_{3}(u) \\
\frac{1}{5} s_{4}(u) \\
s_{5}(u)
\end{array}\right)
$$

As long as $5 t \prod_{j=0}^{4} y_{j} \neq 0$, one can forget this factor since $\left(y_{0}: \ldots: y_{4}\right)$ are given modulo the action of $\mathbb{C}^{*}$.

Notice that the sixth row $r_{5}$ of the augmented matrix of the system:

$$
\left(\begin{array}{llllll}
u_{0}^{5} & u_{1}^{5} & u_{2}^{5} & u_{3}^{5} & u_{4}^{5} & s_{5}(u)
\end{array}\right)
$$

is a linear combination of the first five rows $\left(r_{i}\right)_{i \in\{0, \ldots, 4\}}$ :

$$
r_{5}-s_{1}(u) r_{4}+s_{2}(u) r_{3}-s_{3}(u) r_{2}+s_{4}(u) r_{1}-s_{5}(u) r_{0}=0 .
$$

Thus after setting $u_{i}=0$, one gets 5 equations defining a reduced complete intersection in $\mathbb{P}^{3} \times\left(\mathbb{C}^{*}\right)^{4}$.

Corollary 4.2. Let $y \in X_{t}$ for some $t \in \mathbb{C}$ satisfy

$$
\prod_{j=0}^{4} y_{j} \neq 0 .
$$

Then a line $l \subset \mathbb{P}^{4}$ has contact of order $\geq 5$ with $X_{t}$ at the point $y$ if and only if

$$
l \subset X_{t}
$$


Proof. Looking back at the way equations (4.4) were obtained, one notices that the first $k$ equations in (4.4) define the subset

$$
\left\{\left.(\{l\}, y) \in F(1,2,5)\right|_{\left(\mathbb{C}^{*}\right)^{4}} / 1 \text { has contact of order } \geq k \text { with } X_{t} \text { at } y\right\}
$$

of $\left.F(1,2,5)\right|_{\left(\mathbb{C}^{*}\right)^{4}}$. Thus the corollary rephrases the statement that the 6 -th equation in (4.4) is a combination of the other five.

Theorem 4.3. The inverse image of $\mathcal{H}^{\prime} / \hat{G}$ in $\mathcal{U} / \hat{G}$ is a smooth, irreducible, rational threefold. Thus $\mathcal{H}^{\prime} / \hat{G}$ is a smooth irreducible unirational, therefore rational, surface.

Proof. If $u$ is an element of $\mathbb{P}^{4} \backslash\left(\bigcup_{j \neq k}\left\{u_{j}=u_{k}\right\}\right)$, then the matrix $M(u)$ is invertible. In this case, equation (4.2) can be rewritten as

$$
Y(y)=5 t \prod_{j=0}^{4} y_{j} M\left(u^{i}\right)^{-1} C\left(u^{i}\right) .
$$

Recall that $Y(u)$ is given only in terms of $y^{5}:=\left(y_{0}^{5}: \ldots: y_{4}^{5}\right)$. As long as $t \prod_{j=0}^{4} y_{j} \neq 0$, one may disregard the $t \prod_{j=0}^{4} y_{j}$. As a consequence, equation (4.5) defines a birational transformation

$$
\begin{aligned}
& \gamma^{i}: \mathbb{P}^{3} \rightarrow \mathcal{U} / \hat{G}, \\
& \gamma^{i}(u)=\left(y^{5}, u\right) .
\end{aligned}
$$

If one thinks of $G(2,5)$ as embedded in $\mathbb{P}^{9}$ by the Plücker embedding, then, in terms of the coordinates $\left(\left(y_{j}\right)_{j},\left(u_{j}\right)_{j}\right)$,

$$
p_{i j}=y_{i} y_{j}\left(u_{i}-u_{j}\right) .
$$

So, if $y$ is such that $\prod_{i=0}^{4} y_{i} \neq 0$, then the conditions $y \in l,\{l\} \in \mathcal{H}^{\prime}$ are equivalent to the fact that $M(u)$ is invertible. The map $\gamma^{i}$ defined above is thus a smooth morphism at such pairs $(l,\{y\})$ and therefore the images of these points are smooth in $\mathcal{U} / \hat{G}$. The points of $\mathcal{U}$ standing over these points of $\mathcal{U} / \hat{G}$ are also smooth.

Next we will take a closer look at the lines excluded by the previous corollary:

Theorem 4.4. Let $l \subset X_{t}$ be a line such that $\{l\} \in \mathcal{H} \backslash \mathcal{H}^{\prime}$. Then either $l \subset X_{0}$ or $l$ is a Van Geemen line.

Proof. For symmetry reasons, it is enough to check the proposition for $l$ with $p_{01}=0$. Assume that $l \not \subset X_{0}$. Then one can choose a point $y$ on $l$ such that $\prod_{i=0}^{4} y_{i} \neq 0$. Indeed, one can always do that as long as $l$ is not included in one of the coordinate hyperplanes $H_{i}$. But if $l \subset H_{i}$ and $l \subset X_{t}$ for some $t \in \mathbb{C}$, then $l$ is in the base locus of the pencil $\mathcal{X}$ and thus also in $X_{0}$.

In the proof of Corollary 4.4 it is shown that the condition $p_{01}=0$ becomes $u_{0}=u_{1}$. By considering the map $\psi^{0}$, one may assume

$$
u_{0}=u_{1}=0 \text {. }
$$


The proposition can be proven by analyzing equation (4.2) in this context.

Because $u_{0}=u_{1}=0$, all the other $u_{j}$-s satisfy the following:

$$
u_{j}^{k+3}-s_{1}\left(u^{01}\right) u_{j}^{k+2}+s_{2}\left(u^{01}\right) u_{j}^{k+1}-s_{3}\left(u^{01}\right) u_{j}^{k}=0 .
$$

In particular, for $k=1$ one obtains that the last 4 rows of the matrix $M(u)=M\left(u^{01}\right)$ are linearly dependent and thus, the last 4 rows of $C(u)$ should necessarily satisfy the same dependence relation:

$$
\frac{s_{4}\left(u^{01}\right)}{5}-s_{1}\left(u^{01}\right) \frac{s_{3}\left(u^{01}\right)}{10}+s_{2}\left(u^{01}\right) \frac{s_{2}\left(u^{01}\right)}{10}-s_{3}\left(u^{01}\right) \frac{s_{1}\left(u^{01}\right)}{5}=0 .
$$

Because $s_{4}\left(u^{01}\right)=0$, this relation simplifies to:

$$
\begin{gathered}
s_{2}^{2}\left(u^{01}\right)-3 s_{1}\left(u^{01}\right) s_{3}\left(u^{01}\right)=0 \\
\left(u_{3} u_{4}+\xi u_{2} u_{4}+\xi^{2} u_{2} u_{3}\right)\left(u_{3} u_{4}+\xi^{2} u_{2} u_{4}+\xi u_{2} u_{3}\right)=0
\end{gathered}
$$

where $\xi$ is a primitive third root of unity.

For symmetry reasons it is enough to study the case

$$
u_{3} u_{4}+\xi u_{2} u_{4}+\xi^{2} u_{2} u_{3}=0 .
$$

If two of the $u_{i}$-s are equal, then either all three are equal or those two are actually 0 . If all three are equal, then $l$ must be passing through a point $\left(\mu_{0}:-\mu_{1}: 0: 0: 0\right)$ with $\mu_{0}^{5}=\mu_{1}^{5}=1$, and also through a point $\left(0: 0: x_{2}: x_{3}: x_{4}\right)$, because of the assumption $p_{01}=0$. In this case $l \subset X_{0}$.

Notice also that if any one of the coordinates $\left(u_{2}, u_{3}, u_{4}\right)$ is 0 , then yet another one should be 0 . But it is easy to check that there is no line inside $\mathcal{X}$ passing through a point four of whose coordinates are 0 . Thus one obtains that $u_{2} u_{3} u_{4} \neq 0$. This, together with equation (4.8), justifies a change of coordinates to $v^{01}:=\left(v_{2}: v_{3}: v_{4}\right)$, where

$$
v_{i}=\frac{1}{u_{i}}
$$

Equation (4.8) becomes:

$$
v_{2}+\xi v_{3}+\xi^{2} v_{4}=0
$$

The $u$-terms involved in the algebraic description (4.5) of $\mathcal{U}^{\prime}$ transform as follows under the change $v_{i}=\frac{1}{u_{i}}$ :

$$
s_{k}\left(u^{\prime}\right)=\frac{s_{t o p-k}\left(v^{\prime}\right)}{s_{t o p}\left(v^{\prime}\right)}
$$

where top $=5$ for $u^{\prime}=u$ and $v^{\prime}=v$, top $=4$ for $u^{\prime}=u^{i}$ and $v^{\prime}=v^{i}$ etc.

$$
\delta\left(u^{\prime}\right)=(-1)^{\frac{t o p(t o p-1)}{2}} \frac{\delta\left(v^{\prime}\right)}{s_{t o p}\left(v^{\prime}\right)^{t o p-1}} .
$$

Given $\left(v_{2}: v_{3}: v_{4}\right)$, equations (4.5) well determine $\left(y_{2}^{5}: y_{3}^{5}: y_{4}^{5}\right)$ (up to a common factor). Indeed, after dividing by the $t \prod_{i=0}^{4} y_{i^{-}}$term:

$$
Y^{01}\left(y^{01}\right)=M^{01}\left(u^{01}\right)^{-1} C^{01}\left(u^{01}\right)
$$


where

$$
\begin{aligned}
Y^{01}\left(y^{01}\right):=\left(\begin{array}{c}
y_{2}^{5} \\
y_{3}^{5} \\
y_{4}^{5}
\end{array}\right), C^{01}\left(u^{01}\right):=\left(\begin{array}{c}
s_{1}\left(u^{01}\right) \\
\frac{1}{2} s_{2}\left(u^{01}\right) \\
\frac{1}{2} s_{3}\left(u^{01}\right)
\end{array}\right), \\
M^{01}\left(u^{01}\right):=\left(\begin{array}{ccc}
u_{2} & u_{3} & u_{4} \\
u_{2}^{2} & u_{3}^{2} & u_{4}^{2} \\
u_{2}^{3} & u_{3}^{3} & u_{4}^{3}
\end{array}\right) .
\end{aligned}
$$

Alternatively, following formulas (4.10), and (4.11):

$$
Y^{01}\left(y^{01}\right)=M^{01}\left(v^{01}\right)^{-1} C^{01}\left(v^{01}\right)
$$

with

and the product

$$
C^{01}\left(v^{01}\right)=\frac{1}{s_{3}\left(v^{01}\right)}\left(\begin{array}{c}
s_{2}\left(v^{01}\right) \\
\frac{1}{2} s_{1}\left(v^{01}\right) \\
\frac{1}{2} s_{0}\left(v^{01}\right)
\end{array}\right)
$$

equal to

$$
\delta\left(v^{01}\right) M^{01}\left(v^{01}\right)^{-1}
$$

$$
\left(\begin{array}{ccc}
v_{2}^{3} \delta\left(v^{012}\right) s_{0}\left(v^{012}\right) & -v_{2}^{3} \delta\left(v^{012}\right) s_{1}\left(v^{012}\right) & v_{2}^{3} \delta\left(v^{012}\right) s_{2}\left(v^{012}\right) \\
-v_{3}^{3} \delta\left(v^{013}\right) s_{0}\left(v^{013}\right) & +v_{3}^{3} \delta\left(v^{013}\right) s_{1}\left(v^{013}\right) & -v_{3}^{3} \delta\left(v^{013}\right) s_{2}\left(v^{013}\right) \\
v_{4}^{3} \delta\left(v^{014}\right) s_{0}\left(v^{014}\right) & -v_{4}^{3} \delta\left(v^{014}\right) s_{1}\left(v^{014}\right) & v_{4}^{3} \delta\left(v^{014}\right) s_{2}\left(v^{014}\right)
\end{array}\right)
$$

Thus one derives:

$$
y_{j}^{5}=(-1)^{j} \frac{\delta\left(v^{01 j}\right) v_{j}^{3}}{\delta\left(v^{01}\right) s_{3}\left(v^{01}\right)}\left(s_{2}\left(v^{01}\right)-\frac{1}{2} s_{1}\left(v^{01 j}\right) s_{1}\left(v^{01}\right)+\frac{1}{2} s_{2}\left(v^{01 j}\right)\right)
$$

for $j \in\{2,3,4\}$. Using the identities:

$$
\begin{gathered}
s_{1}\left(v^{01 j}\right)=s_{1}\left(v^{01}\right)-v_{j}, \\
s_{2}\left(v^{01 j}\right)=s_{2}\left(v^{01}\right)-v_{j} s_{1}\left(v^{01}\right)+v_{j}^{2},
\end{gathered}
$$

one can further simplify to

$$
y_{j}^{5}=\frac{(-1)^{j} \delta\left(v^{01 j}\right) v_{j}^{3}}{2 \delta\left(v^{01} s_{3}\left(v^{01}\right)\right.}\left(3 s_{2}\left(v^{01}\right)-s_{1}^{2}\left(v^{01}\right)+v_{j}^{2}\right) .
$$

But formula (4.9) implies $3 s_{2}\left(v^{01}\right)-s_{1}^{2}\left(v^{01}\right)=0$ and then

$$
y_{j}^{5}=\frac{(-1)^{j} \delta\left(v^{01 j}\right) v_{j}^{5}}{2 \delta\left(v^{01}\right) s_{3}\left(v^{01}\right)}
$$

or equivalently,

$$
x_{j}^{5}=\frac{y_{j}^{5}}{v_{j}^{5}}=\frac{(-1)^{j} \delta\left(v^{01 j}\right)}{2 \delta\left(v^{01}\right) s_{3}\left(v^{01}\right)}
$$

for $j \in\{2,3,4\}$. Thus one obtains:

$$
\begin{aligned}
\left(x_{0}^{5}: x_{1}^{5}: x_{2}^{5}: x_{3}^{5}: x_{4}^{5}\right) & =\left(0: 0: v_{4}-v_{3}: v_{2}-v_{4}: v_{3}-v_{2}\right)= \\
= & \left(0: 0: 1: \xi: \xi^{2}\right),
\end{aligned}
$$


since $v^{01} \neq(1: 1: 1)$ lies in the plane $v_{2}+\xi v_{3}+\xi^{2} v_{4}=0$. Then

$$
\left(x_{0}: x_{1}: x_{2}: x_{3}: x_{4}\right)=\left(0: 0: \mu_{2}: \xi^{2} \mu_{3}: \xi \mu_{4}\right)
$$

for some fifth roots of unity $\mu_{2}, \mu_{3}, \mu_{4}$. From here,

$(4.14)\left(y_{2}: y_{3}: y_{4}\right)=\left(v_{2} x_{2}: v_{3} x_{3}: v_{4} x_{4}\right)=\left(v_{2} \mu_{2}: v_{3} \xi^{2} \mu_{3}: v_{4} \xi \mu_{4}\right)$.

In view of formula (4.9), the last statement leads to the following: If $l$ satisfies $p_{01}=0$, then its projection on the last three coordinates:

$$
\begin{aligned}
\mathbb{P}^{1} & \rightarrow \mathbb{P}^{2}, \\
(\alpha: \beta) & \rightarrow\left(\phi_{2}(\alpha, \beta): \phi_{3}(\alpha, \beta): \phi_{4}(\alpha, \beta)\right),
\end{aligned}
$$

is a line of equation

$$
\mu_{2}^{4} z_{2}+\xi^{2} \mu_{3}^{4} z_{3}+\xi \mu_{3}^{4} z_{3}=0
$$

for some fifth roots of unity $\mu_{2}, \mu_{3}, \mu_{4}$, or

$$
\mu_{2}^{4} z_{2}+\xi \mu_{3}^{4} z_{3}+\xi^{2} \mu_{3}^{4} z_{3}=0
$$

if one looks at the other component of the locus of such lines pointed out in formula (4.7). In particular, such a line contains a point with

$$
\left(y_{2}: y_{3}: y_{4}\right)=\left(\mu_{2}: \mu_{3}: \mu_{4}\right) \text {. }
$$

This, together with formula (4.13), are enough to characterize a Van Geemen line.

Putting together the information on smoothness gathered along these 4 sections, one may conclude:

Proposition 4.5. Outside the lines in the base locus of the family $\mathcal{X}, \mathcal{U}$ is a smooth threefold.

\section{The surface Component $\mathcal{S}$ of $\mathcal{H}$}

Putting together the results of Sections 1 through 4, one can prove:

Theorem 5.1. The irreducible components of the relative Hilbert scheme $\mathcal{H}$ are given by:

$$
\mathcal{H}=\mathcal{S} \cup \bigcup_{i=1}^{375} Z_{i}
$$

where $\mathcal{S}$ is a smooth quasi-projective surface proper over $\mathbb{C}$ and for each $i$, $\pi_{\mid Z_{i}}: Z_{i} \rightarrow \mathbb{C}$ is an isomorphism. The Hilbert polynomial of the family $\mathcal{S}$ inside $G(2,5)$ is

$$
p_{t}(n)=500 n-1250 .
$$

Proof. Consider the Plücker embedding $G(2,5) \subset \mathbb{P}^{9}$. Let

$$
T=\mathbb{P}^{9} \backslash\left(\bigcup_{i, j=0}^{4}\left\{p_{i j}=0\right\}\right)
$$

denote the torus in $\mathbb{P}^{9}$ : 
Theorem 4.4 shows that for each $t \neq 0$, the intersection of $\mathcal{H}_{t}$ with the border of the torus $\mathrm{T}$ consists of the 5000 points $\left(2500\right.$ for $\left.t=2 \sqrt[5]{\frac{4}{3}} \mu\right)$, representing the van Geemen lines of the quintic, together with 375 isolated points. Following the description in Section 3, the union of all the Van Geemen points of the relative Hilbert scheme $\mathcal{H}$ gives a set of 500 smooth irreducible curves in $\mathbb{P}^{2} \times \mathbb{C}$ satisfying equations like these:

$$
\left\{\begin{array}{l}
b^{5}+a^{5}-27 c^{5}=0 \\
t b a-6 c^{2}=0
\end{array}\right.
$$

where $(a: b: c)$ are homogeneous coordinates in $\mathbb{P}^{2}$. Each of these curves is a 10-1 cover of $\mathbb{C}$, branched over 0 and $2 \sqrt[5]{\frac{4}{3}} \mu$. From the Propositions 2.2 and 2.3 one gathers that there is only one irreducible 2 -dimensional component $\mathcal{S}$ of $\mathcal{H}$ containing $\mathcal{H}_{0}$ and moreover, this is the only irreducible 2-dimensional component of $\mathcal{H}$ which intersects $\mathcal{H}_{0}$. But since all the irreducible curves of van Geemen points intersect $\mathcal{H}_{0}$, it follows that they are all contained in $\mathcal{S}$.

On the other hand, as a result Section 4 , the preimage $\mathcal{H}^{\prime}$ of $G(2,5) \cap T$ in $\mathcal{H}$ is also irreducible. Indeed, Theorem 4.3 states that $\mathcal{H}^{\prime} / \hat{G}$ is irreducible; a simple exercise starting from equations (4.5) shows that $\mathcal{H}^{\prime}$ itself is irreducible. One may also find a further verification of this fact in formula (6.6), which explicitly describes the map $\gamma^{i}$.

Theorem 4.4 shows that $\mathcal{H} \backslash \mathcal{H}^{\prime}$ consists of the lines in the base locus of $\mathcal{X}$, plus those in $\mathcal{H}_{0}$ and the van Geemen lines, contained, as seen above, in $\mathcal{S}$. Form the structure of $\mathcal{H}$ :

$$
\mathcal{H}=\mathcal{S} \cup \bigcup_{i=1}^{375} Z_{i}
$$

where $\mathcal{S}$ is a smooth quasi-projective surface proper over $\mathbb{C}$ and for each $i$, $Z_{i}$ corresponds to some line in the base locus of $\mathcal{X}$, so $\pi_{\mid Z_{i}}: Z_{i} \rightarrow \mathbb{C}$ is an isomorphism.

The Hilbert polynomial of the family $\left(\mathcal{S}_{t}\right)_{t}$ can be computed as

$$
p(n)=2 p_{\mathcal{H}} \text { red }(n)=2(50(5 n-5)-375)=500 n-1250
$$

since each $C_{i, j, \mu}$ is embedded in $\mathrm{G}(2,5)$ as a degree 5 plane curve, each of the 375 base-locus points is at the intersection of exactly 2 of the components of $\mathcal{H}_{0}$ red, with intersection multiplicity 1 , while the whole $\mathcal{H}_{0}$ red is embedded in $\mathcal{H}$ with embedding multiplicity 2 .

Theorem 5.2. The quotient $\mathcal{S} / \hat{G}$ of the surface $\mathcal{S}$ is a smooth, irreducible, quasi-projective rational surface, proper over $\mathbb{C}$.

Proof. The finite group $\hat{G}$ acts on the smooth surface $\mathcal{S}$. One needs to check whether the quotients of those points having non-trivial isotropy groups under this action are smooth points of $\mathcal{S} / \hat{G}$. First recall from Theorem 4.3 that the open subset $\mathcal{H}^{\prime} / \hat{G}$ of $\mathcal{S} / \hat{G}$ is smooth and rational. The complement of $\mathcal{H}^{\prime}$ in $\mathcal{S}$ consists of the van Geemen lines and the lines in $\mathcal{H}_{0}$. Each of 
the van Geemen lines has a trivial isotropy group, as can be easily seen from their definition. Consider now a line $l_{1}$ in the base locus of the family $\mathcal{X}$, and a line $l_{2}$ contained in $X_{0}$, but not in the base locus. The point $\left\{l_{2}\right\} \in \mathcal{H}_{0}$ has order 5 isotropy group $G_{2}$ : if, for example, $l_{2}$ passes through $(1:-1: 0: 0: 0)$ and $\left(0: 0: x_{2}: x_{3}: x_{4}\right) \in \mathbb{P}^{4}$, then

$$
G_{2}=\left\{(1: 1: \mu: \mu: \mu) / \mu^{5}=1\right\} .
$$

The point $\left\{l_{1}\right\} \in \mathcal{H}_{0}$ has order 25 isotropy group $G_{1}$ : if, for example, $l_{1}$ passes through $(1:-1: 0: 0: 0)$ and $(0: 0: 1:-1: 0) \in \mathbb{P}^{4}$, then

$$
G_{1}=\left\{\left(1: 1: \mu_{1}: \mu_{1}: \mu_{2}\right) / \mu_{1}^{5}=1, \mu_{2}^{5}=1\right\},
$$

with two generators $(1: 1: \mu: \mu: \mu)$ and $(\mu: \mu: 1: 1: \mu)$. Each of these generators gives a pseudoreflection of $\mathcal{S}$, i.e. the set of points in $\mathcal{S}$ fixed by it is a divisor of $\mathcal{S}$. Indeed, the set of points in $\mathcal{S}$ fixed by $(1: 1: \mu: \mu: \mu)$ corresponds to the lines in the cones $C_{0,1, \nu}$, of vertices

$$
(1:-\nu: 0: 0: 0)
$$

$\nu^{5}=1$, over the curve

$$
\left\{\left(0: 0: x_{2}: x_{3}: x_{4}\right) / x_{2}^{5}+x_{3}^{5}+x_{4}^{5}=0\right\} .
$$

Following [ST, the above property is sufficient for the images of $l_{1}$ and $l_{2}$ to be smooth points of $\mathcal{S} / \hat{G}$. Thus $\mathcal{S} / \hat{G}$ is smooth everywhere.

\section{Properties of the fiber $(\mathcal{S} / \hat{G})_{w}$}

We are now ready to prove:

Proposition 6.1. The family $(\mathcal{S} / \hat{G})$ has double fiber at $w=0, \frac{2^{7}}{5}$.

The fiber $(\mathcal{S} / \hat{G})_{w}$ at $w \neq 0, \frac{2^{7}}{5}$ consists of two isomorphic connected curves.

Proof. Recall from Corollary 4.3 that the following rational map

$$
\hat{\psi}^{i}: \mathcal{U} / \hat{G} \rightarrow \mathbb{P}^{3}
$$

is birational. Let $U_{w}^{i}$ denote the closure of $\hat{\psi}^{i}\left((\mathcal{U} / \hat{G})_{w}\right)$. Starting from formula (4.5), we will derive the equation of the surface $U_{w}^{i}$ in $\mathbb{P}^{3}$ and then will prove that $U_{w}^{i}$ has two irreducible surface components.

First, one can explicitly write down the inverse $\gamma^{i}$ of the above map. Although in this context the $i$-th coordinate $u_{i}=0$, we will treat it here as all the other coordinates, for symmetry purposes. If $u_{j} \neq u_{k}$ for all $j, k \in\{0, \ldots, 4\}$, the matrix $M^{-1}(u)$ has elements

$$
M^{-1}(u)_{j k}=(-1)^{j+k} \frac{\delta\left(u^{j}\right)}{\delta(u)} s_{4-k}\left(u^{j}\right)
$$


where $j, k \in\{0, \ldots, 4\}$. (Indeed, starting from the identity

$$
\sum_{k=0}^{4}(-1)^{k} s_{4-k}\left(u^{j}\right) u_{l}^{k}=\prod_{h \neq j}\left(u_{l}-u_{h}\right)=\delta_{j l}(-1)^{j} \frac{\delta(u)}{\delta\left(u^{j}\right)},
$$

one finds that

$$
\sum_{k=0}^{4} M^{-1}(u)_{j k} M(u)_{k l}=\sum_{k=0}^{4}(-1)^{j+k} \frac{\delta\left(u^{j}\right)}{\delta(u)} s_{4-k}\left(u^{j}\right) u_{l}^{k}=\delta_{j l},
$$

where $\delta_{j l}$ is the Kröenecker delta symbol.)

By formula (4.5), $\gamma^{i}$ is given by

$$
y_{j}^{5}=\sum_{k=0}^{4} M^{-1}(u)_{j k} C(u, y)_{k}
$$

so

$$
y_{j}^{5}=\left(5 t \prod_{l=0}^{4} y_{l}\right) \frac{\delta\left(u^{j}\right)}{\delta(u)} \sum_{k=0}^{4} \frac{(-1)^{j+k}}{\left(\begin{array}{l}
5 \\
k
\end{array}\right)} s_{4-k}\left(u^{j}\right) s_{k}(u) .
$$

Use the identity

$$
s_{k}(u)=s_{k}\left(u^{j}\right)+u_{j} s_{k-1}\left(u^{j}\right)
$$

to further simplify:

$$
\begin{aligned}
y_{j}^{5} & =\left(5 t \prod_{l=0}^{4} y_{l}\right) \frac{\delta\left(u^{j}\right)}{\delta(u)} \sum_{k=0}^{4} \frac{(-1)^{j+k}}{\left(\begin{array}{c}
5 \\
k
\end{array}\right)} s_{4-k}\left(u^{j}\right)\left(s_{k}\left(u^{j}\right)+u_{j} s_{k-1}\left(u^{j}\right)\right)= \\
& =\left(5 t \prod_{l=0}^{4} y_{l}\right) \frac{\delta\left(u^{j}\right)}{\delta(u)} \frac{(-1)^{j}}{10}\left(s_{2}^{2}\left(u^{j}\right)-3 s_{1}\left(u^{j}\right) s_{3}\left(u^{j}\right)+12 s_{4}\left(u^{j}\right)\right) .
\end{aligned}
$$

Notation . Let $g\left(u^{j}\right):=s_{2}^{2}\left(u^{j}\right)-3 s_{1}\left(u^{j}\right) s_{3}\left(u^{j}\right)+12 s_{4}\left(u^{j}\right)$.

The following lemma can be verified by direct computations:

Lemma 6.2. Let $\{h, i, j, k, l\}=\{0, \ldots, 4\} . g\left(u^{i}\right)$ has the following proprieties:

$$
\begin{gathered}
\left.g\left(u^{i}\right)\right|_{\left\{u_{j}=u_{i}\right\}}=\left.g\left(u^{j}\right)\right|_{\left\{u_{j}=u_{i}\right\}} \\
\left.g\left(u^{i}\right)\right|_{\left\{u_{j}=u_{k}\right\}}=\left(u_{l}-u_{j}\right)^{2}\left(u_{h}-u_{j}\right)^{2} \\
\left.\frac{\partial g\left(u^{i}\right)}{\partial u_{j}}\right|_{\left\{u_{j}=u_{k}\right\}}=\left(u_{l}-u_{j}\right)\left(u_{h}-u_{j}\right)\left(u_{l}+u_{h}-2 u_{j}\right) \\
\frac{\partial^{2} g\left(u^{i}\right)}{\partial u_{j}^{2}}=\frac{\partial^{2} g\left(u^{j}\right)}{\partial u_{i}^{2}}=2 \sum_{k \in\{0, . ., 4\} \backslash\{i, j\}}\left(u_{h}-u_{k}\right)\left(u_{l}-u_{k}\right)
\end{gathered}
$$




$$
\left.\frac{\partial^{2} g\left(u^{i}\right)}{\partial u_{j}^{2}}\right|_{\left\{u_{k}=u_{l}\right\}}=2\left(u_{h}-u_{k}\right)^{2}
$$

To recap, we have shown that the inverse $\gamma^{i}$ of $\hat{\psi}^{i}$ is given by:

$$
y_{j}^{5}=5 t\left(\prod_{l=0}^{4} y_{l}\right) \frac{\delta\left(u^{j}\right)}{\delta(u)} \frac{(-1)^{j}}{10} g\left(u^{j}\right) .
$$

By taking product of the equations (6.6) when $j$ varies from 0 to 4 and then simplifying the $\prod_{j=0}^{4} y_{j}^{5}$-factor, one finds the pullback by $\gamma^{i}$ of the equation for $(\mathcal{U} / \hat{G})_{w}$, on the set $\mathbb{P}^{3} \backslash\left(\bigcup_{i \neq j}\left\{u_{i}=u_{j}\right\}\right)$ :

$$
1=\frac{w}{2^{5}} \prod_{j=0}^{4} \frac{\delta\left(u^{j}\right)}{\delta(u)} g\left(u^{j}\right),
$$

where $w=t^{5}$. After simplification, here is the equation of the surface $U_{w}^{i}$ in $\mathbb{P}^{3}$ :

$$
\delta^{2}(u)=\frac{w}{2^{5}} \prod_{j=0}^{4} g\left(u^{j}\right)
$$

Notation . Let

$$
G(u):=\prod_{j=0}^{4} g\left(u^{j}\right)
$$

To prove the existence of two irreducible components of $U_{w}^{i}$, we will find a symmetric, homogeneous degree 10 polynomial $P(u)$ such that

$$
\prod_{j=0}^{4} g\left(u^{j}\right)-\frac{3}{4} \delta^{2}(u)=P^{2}(u) .
$$

Equations (6.7) and (6.8) will thus show that the fiber at $w=\frac{2^{7}}{3}$ appears with multiplicity 2 in the family $\left((\mathcal{U} / \hat{G})_{w}\right)_{w}$. Also, equation (6.7) may be rewritten as

$$
\left(1-\frac{3 w}{2^{7}}\right) \delta^{2}(u)-\frac{w}{2^{5}} P^{2}(u)=0,
$$

which factors as the product of two equations for each $w \in \mathbb{C}$. Moreover, for generic $w$, each of these equations define an irreducible surface in $\mathbb{P}^{3}$.

In order to write $P(u)$ explicitly, one can consider $\frac{G(u)}{\delta^{2}(u)}$ as a rational function in the variable $u_{0}$ and decompose it into a sum of simple fractions:

$$
\frac{G(u)}{\delta^{2}(u)}=\sum_{i=1}^{4} \frac{A_{i}\left(u^{0}\right)}{\left(u_{0}-u_{i}\right)^{2}}+\sum_{i=1}^{4} \frac{B_{i}\left(u^{0}\right)}{\left(u_{0}-u_{i}\right)}+C\left(u^{0}\right),
$$


with coefficients satisfying:

$$
\begin{aligned}
& A_{i}\left(u^{0}\right)=\left.\frac{1}{\delta^{2}\left(u^{0}\right) \prod_{j \neq 0, i}\left(u_{i}-u_{j}\right)^{2}} G(u)\right|_{u_{0}=u_{i}}, \\
& B_{i}\left(u^{0}\right)+A_{i}\left(u^{0}\right) \sum_{j \neq 0, i} \frac{2}{u_{i}-u_{j}}=\left.\frac{1}{\delta_{0}^{2} \prod_{j \neq 0, i}\left(u_{i}-u_{j}\right)^{2}} \frac{\partial G(u)}{\partial u_{0}}\right|_{u_{0}=u_{i}} \\
& C\left(u^{0}\right)=\frac{1}{2^{4} \delta^{2}\left(u^{0}\right)} g\left(u^{0}\right) \prod_{i=1}^{4} \frac{\partial^{2} g\left(u^{i}\right)}{\partial u_{0}^{2}}
\end{aligned}
$$

Using properties (6.1) - (6.3) in lemma 6.2, one finds:

$$
A_{i}\left(u^{0}\right)=\frac{g^{2}\left(u^{0}\right)}{\delta^{2}\left(u^{0 i}\right)} \text { and } B_{i}\left(u^{0}\right)=\frac{1}{\delta_{0 i}^{2}} g\left(u^{0}\right) \frac{\partial g\left(u^{0}\right)}{\partial u_{i}} .
$$

Let $a_{i}\left(u^{0}\right)=\frac{g\left(u^{0}\right)}{\delta\left(u^{0 i}\right)}$. Next one checks that

$$
\begin{gathered}
\frac{G(u)}{\delta^{2}(u)}-\frac{3}{4}=\sum_{i=1}^{4} \frac{A_{i}\left(u^{0}\right)}{\left(u_{0}-u_{i}\right)^{2}}+\sum_{i=1}^{4} \frac{B_{i}\left(u^{0}\right)}{\left(u_{0}-u_{i}\right)}+C\left(u^{0}\right)-\frac{3}{4}= \\
=\left(\sum_{i=1_{, 4}^{-}} \frac{a_{i}\left(u^{0}\right)}{\left(u_{0}-u_{i}\right)}+E\left(u^{0}\right)\right)^{2}
\end{gathered}
$$

where

$$
E\left(u^{0}\right)=E_{i}\left(u^{0}\right)=\frac{B_{i}\left(u^{0}\right)}{2 a_{i}\left(u^{0}\right)}-\sum_{j \neq 0, i} \frac{a_{j}\left(u^{0}\right)}{\left(u_{i}-u_{j}\right)}
$$

does not depend on $i$ and

$$
E^{2}\left(u^{0}\right)=C\left(u^{0}\right)-\frac{3}{4}
$$

Indeed, one can verify the last two properties by again decomposing into sums of simple fractions in new variables $u_{i}$. It is enough to do it for $i=1$ :

$$
\begin{aligned}
& E_{1}\left(u^{0}\right)=\frac{1}{2 \delta\left(u^{01}\right)} \frac{\partial g\left(u^{0}\right)}{\partial u_{1}}-\sum_{j=2}^{4} \frac{g\left(u^{0}\right)}{\delta\left(u^{0 j}\right)\left(u_{1}-u_{j}\right)}= \\
& =\frac{1}{\delta\left(u^{01}\right)}\left(\frac{1}{2} \frac{\partial g\left(u^{0}\right)}{\partial u_{1}}-\sum_{j=2}^{4} \frac{g\left(u^{0}\right)\left(u_{i}-u_{j}\right)\left(u_{k}-u_{j}\right)}{\left(u_{1}-u_{2}\right)\left(u_{1}-u_{3}\right)\left(u_{1}-u_{4}\right)}\right)= \\
& =\frac{1}{\delta\left(u^{01}\right)}\left(\frac{1}{2} \frac{\partial g\left(u^{0}\right)}{\partial u_{1}}-\frac{g\left(u^{0}\right)}{2\left(u_{1}-u_{2}\right)\left(u_{1}-u_{3}\right)\left(u_{1}-u_{4}\right)} \frac{\partial^{2} g\left(u^{0}\right)}{\partial u_{1}^{2}}\right)= \\
& =\frac{1}{\delta\left(u^{01}\right)}\left(\frac{1}{2} \frac{\partial g\left(u^{0}\right)}{\partial u_{1}}-\sum_{j=2}^{4} \frac{\left(u_{i}-u_{j}\right)\left(u_{k}-u_{j}\right)}{2\left(u_{1}-u_{j}\right)} \frac{\partial^{2} g\left(u^{0}\right)}{\partial u_{1}^{2}}\right)
\end{aligned}
$$


after using properties (6.4) and (6.2). (Here $\{i, j, k\}=\{2,3,4\})$. On the other hand, writing

$$
C\left(u^{0}\right)=\frac{1}{2^{4} \delta^{2}\left(u^{0}\right)} g\left(u^{0}\right) \prod_{i=1}^{4} \frac{\partial^{2} g\left(u^{i}\right)}{\partial u_{0}^{2}}
$$

as a sum of simple fractions in the variable $u_{1}$ and using Lemma 6.2, one obtains the following:

$$
\begin{aligned}
& C\left(u^{0}\right)=\frac{1}{\delta^{2}\left(u^{01}\right)}\left(\frac{1}{2} \frac{\partial g\left(u^{0}\right)}{\partial u_{1}}-\sum_{j=2}^{4} \frac{\left(u_{i}-u_{j}\right)\left(u_{k}-u_{j}\right)}{2\left(u_{1}-u_{j}\right)} \frac{\partial^{2} g\left(u^{0}\right)}{\partial u_{1}^{2}}\right)^{2}+\frac{3}{4}= \\
& =E_{1}\left(u^{0}\right)^{2}+\frac{3}{4} .
\end{aligned}
$$

Since this works equally well for each $E_{i}\left(u^{0}\right)$, we have proven formula (6.13) which in turn implies

$$
\frac{G(u)}{\delta^{2}(u)}=\left(\sum_{i=1}^{4} \frac{g\left(u^{0}\right)}{\delta\left(u^{0 i}\right)\left(u_{0}-u_{i}\right)}+E\left(u^{0}\right)\right)^{2}+\frac{3}{4} .
$$

Thus formula (6.8) is proven.

We now know that for $w$ generic, the fiber $(\mathcal{U} / \hat{G})_{w}$ has two irreducible components. Going back to $(\mathcal{S} / \hat{G})_{w}$, notice that the two corresponding irreducible components must be disjoint. Indeed, formula (6.8) shows that the points of intersection of the above two components, if existent, should be among the van Geemen lines. This would contradict Lemma 3.1 which states that for $t \neq \mu \sqrt[5]{\frac{2^{7}}{3}}$, the van Geemen lines define smooth points of $\mathcal{S}_{t}$. Also, a simple check shows that the group $\hat{G}$ acts freely on the set of these lines and they also give smooth points of $(\mathcal{S} / \hat{G})_{w}$ (see also Lemma 6.3). Thus for $w \neq 0, \frac{2^{7}}{3},(\mathcal{S} / \hat{G})_{w}$ has (at least) two connected components.

More information on the fiber $(\mathcal{S} / \hat{G})_{w}$ can be derived by studying the action of the group $\hat{G}$ on $\mathcal{S}$. One can decompose this action into the action of $G$, which fixes the fiber, and that of $\mu=\prod_{j} \mu_{j}$ on $t \in \mathbb{C}$. The group of fifth roots of unity acts freely on $(\mathbb{C})^{*}$. The smoothness of the generic $(\mathcal{S} / G)_{t}$ follows via the following lemma:

Lemma 6.3. Let the group $G$ act on the projective space $\mathbb{P}^{4}$ with coordinates $\left(x_{0}: \ldots: x_{4}\right)$. Then the only lines in $\mathbb{P}^{4}$ with nontrivial isotropy group are those inside the hyperplanes $H_{i}=\left(x_{i}=0\right)$.

Proposition 6.4. For $t$ generic, $\mathcal{H}_{t} / G$ consists of two isomorphic smooth hyperelliptic genus 6 curves together with 15 isolated points.

Proof. By the preceding lemma, $G$ acts freely on $\mathcal{S}_{t}$ for all $t \neq 0$. Thus the generic quotient $\mathcal{S}_{t} / G$ consists of two smooth curves of genus 6 , as may be 
computed by the Hurwitz formula. Each of the 375 isolated points has an isotropy group of order 5 , thus one obtains 15 isolated points in the quotient.

One can see that each component $C_{t}^{1}, C_{t}^{2}$ of the generic fiber $\mathcal{S}_{t} / G$ is hyperelliptic by examining the quotients of the Van Geemen points. There are 5000 such points, organized in 10 families of 500. Each such family is the intersection of $\mathcal{S}_{t}$ with a hyperplane $\left(p_{i j}=0\right)$ in $\mathbb{P}^{9}$ with Plücker coordinates. On the other hand, formula (6.8) shows that the action of $\tau \in \mathbb{Z}_{2}$ on $\mathcal{S}_{t} / G$, induced by the interchanging of two coordinates in $\mathbb{P}^{4}$, is mapping isomorphically one component into the other, so the 500 points in each family split into two families of 250. Each of these families corresponds to the linear divisor given by the hyperplane section of the corresponding component in $\mathbb{P}^{9}$. After quotienting by the $\mathrm{G}$-action, one obtains 10 families of 2 points, and linear equivalence is preserved. The linear system $\left|p_{1}+p_{2}\right|$ in $C_{t}^{i}$, where $p_{1}, p_{2}$ are the classes of the lines through:

$$
\left(\begin{array}{c}
\left(1: \xi: \xi^{2}: 0: 0\right) \\
(1: 1: 1: a: b)
\end{array}\right) \text { and }\left(\begin{array}{c}
\left(1: \xi^{2}: \xi: 0: 0\right) \\
(1: 1: 1: b: a)
\end{array}\right)
$$

is base-point-free and gives a 2-1 morphism $q_{t}^{i}$ into $\mathbb{P}^{1}$. Moreover, since $a$ and $b$ above depend holomorphically on $t$, it follows that the morphism $q_{t}^{i}$ does also. Notice also that even if $C_{t}^{i}$ is singular, one may still repeat the above argument for its normalization, since the Van Geemen points are smooth for $t \neq 2 \sqrt[5]{\frac{4}{3}} \mu$. This even works for $t=2 \sqrt[5]{\frac{4}{3}} \mu$, since the Van Geemen points there are smooth points of the reduced fiber. Thus one establishes a rational map from $\mathcal{S} / G$ into $\mathbb{P}^{1}, 2-1$ on fibers.

Remark 6.5. By Hurwitz formula, the irreducible components of $\mathcal{H}_{0} / \hat{G}$ are all rational. Indeed, with the notations of section 1 and Theorem 5.2, there is an order 5 subgroup of $\hat{G}$ permuting the cones $\left(C_{i, j, \mu}\right)_{\mu}$, another order 5 subgroup fixing each element of $C_{i, j, \mu}$, and from the order 25 remaining action:

$$
2 \cdot(6-1)=25 \cdot 2(0-1)+15 \cdot 4 .
$$

Since there are 10 such irreducible components of $\mathcal{H}_{0} / \hat{G}$, intersecting two by two at 15 points, one immediately gets a total genus of 6 for the reduced $\mathcal{H}_{0} / \hat{G}$.

\section{Properties of the fibers $\mathcal{S}_{t}$}

Proposition 7.1. The fiber of the family $\left(\mathcal{S}_{t}\right)_{t}$ at $t \neq 0,2 \sqrt[5]{\frac{4}{3}} \mu$ consists of two isomorphic connected curves of genus 626 and degree 250 in $G(2,5)$. The fibers at $t=0$ and $2 \sqrt[5]{\frac{4}{3}} \mu$ are connected and are contained in the family with multiplicity 2. The set of all the connected curves in the family can be parametrized by a quasi-projective curve which extends to a genus 2 hyperelliptic curve $C_{2}$. 
Proof. Proposition 6.1 shows that for $w \neq 0, \frac{2^{7}}{3},(\mathcal{S} / \hat{G})_{w}$ consists of two disjoint isomorphic connected curves, so the Stein factorization of the morphism

$$
(\mathcal{S} / \hat{G}) \stackrel{\pi / \hat{G}}{\longrightarrow}(\mathbb{C} / \hat{G})
$$

is

$$
(\mathcal{S} / \hat{G}) \stackrel{\pi_{2} / \hat{G}}{\longrightarrow}\left(C_{2} / \hat{G}\right) \stackrel{\beta / \hat{G}}{\longrightarrow}(\mathbb{C} / \hat{G}),
$$

where $\beta / \hat{G}$ extends to a double cover of $\mathbb{P}^{1}$ branched at 0 and $\frac{2^{7}}{3}$.

A similar statement is true for the fibers $\mathcal{S}_{t}$. The fact that the $\hat{G}$-action does not influence the number of connected components of the generic fiber may be checked in different ways: either by recalling the behavior of $\mathcal{H}$ in a neighborhood of $\mathcal{H}_{0}$ described in Section 2, or computationally be checking that the equations (6.6) are in general irreducible.

Consider the closure $\overline{\mathcal{S}}$ of the quasi-projective surface $\mathcal{S}$ in $G(2,5) \times \mathbb{P}^{1}$. Let $\tilde{\mathcal{S}}$ be the normalization of $\overline{\mathcal{S}}$. By Stein factorization theorem, the morphism

$$
\tilde{\mathcal{S}} \stackrel{\tilde{\pi}}{\longrightarrow} \mathbb{P}^{1}
$$

factors as

$$
\tilde{\mathcal{S}} \stackrel{\tilde{\pi}_{2}}{\longrightarrow} C_{2} \stackrel{\bar{\beta}}{\longrightarrow} \mathbb{P}^{1},
$$

where $\tilde{\pi}_{2}$ is a projective morphism with connected fibers and $\bar{\beta}$ is a $2-1$ morphism, ramified over 0 and $2 \sqrt[5]{\frac{4}{3}} \mu$, for $\mu^{5}=1$, while the curve $C_{2}$ must be a hyperelliptic curve of genus 2 .

\section{The Stable Limits $\tilde{\mathcal{S}}_{\infty}$ AND $\tilde{\mathcal{S}}_{\infty} / \hat{G}$}

Consider the following two compactifications of the surface $\mathcal{S}$ at $t=\infty$ :

First, let $\overline{\mathcal{S}}$ be the closure of the quasi-projective surface $\mathcal{S} \subset G(2,5) \times \mathbb{C}$ in $G(2,5) \times \mathbb{P}^{1}$. This surface is in fact not normal and the fiber $\overline{\mathcal{S}}_{\infty}$ has some embedded points, as Lemma 8.2 will show.

Second, let $\tilde{G}(2,5)$ denote the blow-up of the Grassmannian $G(2,5)$ of lines in $\mathbb{P}^{4}$, along the subvarieties $G_{i}(2,4)$ parametrizing lines inside the coordinate hyperplanes of $\mathbb{P}^{4}$. Let $\tilde{\mathcal{S}}$ be the closure of $\mathcal{S}$ in $\tilde{G}(2,5) \times \mathbb{P}^{1}$. The surface $\tilde{\mathcal{S}}$ turns out to be smooth, as will be seen in Theorem 8.3. The fiber $\tilde{\mathcal{S}}_{\infty}$ is the stable limit of the family $\mathcal{S}$ at $t=\infty$ and consists of two connected components $\tilde{\mathcal{C}}^{\xi}$ and $\tilde{\mathcal{C}}^{\xi^{2}}$, which are isomorphic and reducible:

$$
\tilde{\mathcal{C}}^{\xi}=\bigcup_{i=0}^{4} \tilde{\mathcal{C}}_{i}^{\xi},
$$

with $\tilde{\mathcal{C}}_{i}^{\xi}$ smooth isomorphic curves intersecting pairwise transversely at 25 points. 
The core of the argument here will consist in understanding the structure of the morphism $p: \tilde{G}(2,5) \rightarrow G(2,5)$ and the way the image of $\overline{\mathcal{S}}$ in $G(2,5)$ transforms under $p$ :

Set $\{h, i, j, k, l\}=\{0, \ldots, 4\}$. Working on the affine subsets $\mathbb{A}^{6} \cong U_{i j} \subset$ $G(2,5), U_{i j}=\left(p_{i j} \neq 0\right)$, with coordinates $\left(x_{k}, x_{l}, x_{h}, y_{k}, y_{l}, y_{h}\right)$, one sees that blowing-up the ideal

$$
\left(x_{k}, y_{k}\right) \cdot\left(x_{l}, y_{l}\right) \cdot\left(x_{h}, y_{h}\right)
$$

of $\left(\bigcup_{i=0}^{4} G_{i}(2,4)\right) \cap V_{i j}$ in $V_{i j}$ amounts to taking product of three separate blow-ups, in the directions $\left(x_{k}, y_{k}\right),\left(x_{l}, y_{l}\right)$, and $\left(x_{h}, y_{h}\right)$ :

$$
\left(B l_{(0,0)} \mathbb{A}^{2}\right) \times\left(B l_{(0,0)} \mathbb{A}^{2}\right) \times\left(B l_{(0,0)} \mathbb{A}^{2}\right) \longrightarrow \mathbb{A}^{6}
$$

The fibers contained in the exceptional divisors are:

- $\mathbb{P}^{1}$ over points of $G_{i}(2,4) \backslash\left(\bigcup_{j \neq i} G_{i j}(2,3)\right)$, where each $G_{i j}(2,3)$ denotes the Grassmannian of lines in the plane $\left(x_{i}=x_{j}=0\right)$

- $\mathbb{P}^{1} \times \mathbb{P}^{1}$ over points of $G_{i j}(2,3)$ except for those corresponding to the lines $\left(x_{i}=x_{j}=x_{k}=0\right)$

- $\mathbb{P}^{1} \times \mathbb{P}^{1} \times \mathbb{P}^{1}$ over the points of $G(2,5)$ corresponding to lines $\left(x_{i}=\right.$ $\left.x_{j}=x_{k}=0\right)$.

Furthermore, if one identifies $\overline{\mathcal{S}}$, respectively $\tilde{\mathcal{S}}$ with their images in $G(2,5)$ and $\tilde{G}(2,5)$, one will obtain that

$$
p^{*}(\overline{\mathcal{S}})=\tilde{\mathcal{S}} \cup\left(\bigcup_{i=0}^{4} E_{i}\right)
$$

scheme-theoretically, where $E_{i}$ are the exceptional divisors in $\tilde{G}(2,5)$. This allows for a simple algebraic presentation of $\tilde{\mathcal{S}}$, to the effect that $\tilde{\mathcal{S}}$ is a smooth surface.

The action of the group $\hat{G}$ on $G(2,5)$ extends canonically to an action on $\tilde{G}(2,5)$, making the morphism

$$
p: \tilde{G}(2,5) \rightarrow G(2,5)
$$

$\hat{G}$-equivariant. A brief study of this action will show that $\tilde{\mathcal{S}} / \hat{G}$ is a smooth surface and the stable limit at $w=\infty$ of the family $\mathcal{S} / \hat{G}$ is

$$
\tilde{\mathcal{S}}_{\infty} / \hat{G}=(\tilde{\mathcal{C}} \xi / \hat{G}) \bigcup\left(\tilde{\mathcal{C}}^{\xi^{2}} / \hat{G}\right)
$$

where $\tilde{\mathcal{C}}^{\xi} / \hat{G}$ is the union of 5 smooth rational curves intersecting pairwise transversely.

Here we will keep the notations introduced at the beginning of section 1 .

In AK2, the subset $I$ of $\mathrm{G}(2,5)$ consisting of all the lines incident to the 5 components of the base locus $\mathcal{B}$ is described as a complete intersection 
surface in $\mathrm{G}(2,5)$ given by the following equations:

$$
\sum_{j \neq i ; j=0}^{4} p_{i j}^{5}=0
$$

for $i=0,4$. One of these five equations is a linear combination of the others, which amounts to saying that whenever a line $l$ intersects 4 of the components $B_{i}$, it automatically intersects the fifth.

According to the possible multiplicities of intersection of a line $l$ with the various $B_{i}$-s, one distinguishes the following irreducible components of $I$ :

(1) 50 components, each of which consists of lines in a cone over one of the $B_{l}$-s and having as vertex one of the points in $B_{i j k}$;

(2) 15 components, each of which consists of lines intersecting two of the curves $B_{i j}$ and $B_{k l}$;

(3) 10 components, each of which consists of lines intersecting one $B_{i j}$ and $B_{k}$ and $B_{l}$ and $B_{h}$ (but in general not $B_{k l}, B_{k h}$ or $B_{l h}$, nor $B_{i j k} B_{i j l}$ or $\left.B_{i j h}\right)$;

(4) The image of $\overline{\mathcal{S}} \in G(2,5) \times \mathbb{P}^{1}$ through projection on the Grassmannian $G(2,5)$.

We will start with a description of $\overline{\mathcal{S}}$ initiated by $\mathrm{AK} 2$ :

Proposition 8.1. $\overline{\mathcal{S}}$ is isomorphic onto its image in $G(2,5)$, which is the closure of the set

$$
\left\{\{l\} \in G(2,5) / l \cap B_{i} \neq \emptyset, l \cap B_{i j}=\emptyset, \forall i, j \in\{0, \ldots 4\}, i \neq j\right\} .
$$

Proof. Indeed, if one considers the line $l$ embedded as $\phi: \mathbb{P}^{1} \rightarrow \mathbb{P}^{4}$, then $\{l\} \in \mathcal{H}$ if and only if the pull-back $\phi^{*} f$ of the rational map on $\mathbb{P}^{4}$

$$
f\left(\left(x_{0}: \ldots: x_{4}\right)\right)=\frac{x_{0}^{5}+x_{1}^{5}+x_{2}^{5}+x_{3}^{5}+x_{4}^{5}}{x_{0} x_{1} x_{2} x_{3} x_{4}}
$$

is a constant map, i.e. $\phi^{*}\left(\mathcal{O}_{\mathbb{P}^{4}}\left(X_{0}-\sum_{i=0}^{4} H_{i}\right) \cong \mathcal{O}_{\mathbb{P}^{1}}\right.$. Hence

$$
\left\{\{l\} \in G(2,5) / l \cap B_{i} \neq \emptyset, l \cap B_{i j}=\emptyset, \forall i, j \in\{0, \ldots 4\}, i \neq j\right\} \subset \overline{\mathcal{S}},
$$

and on the other hand $\overline{\mathcal{S}}$ is contained in the variety $I$ of all the lines intersecting the $B_{i}$-s. Since $\overline{\mathcal{S}}$ is irreducible, it is the closure of the above open set.

Lemma 8.2. The reduced structure of $\overline{\mathcal{S}}_{\infty}$ is given by:

$$
\overline{\mathcal{S}}_{\text {ored }}=\bigcup_{i=0}^{4}\left(\mathcal{C}_{i}^{\xi} \cup \mathcal{C}_{i}^{\xi^{2}}\right)
$$

where all $\mathcal{C}_{i}^{\xi}$ and $\mathcal{C}_{i}^{\xi^{2}}$ are smooth irreducible curves of genus 76, and they intersect pairwise transversely as follows: for each $i, j \in\{0, \ldots, 4\}$,

$$
\mathcal{C}_{i}^{\xi} \cap \mathcal{C}_{i}^{\xi^{2}} \cap \mathcal{C}_{j}^{\xi} \cap \mathcal{C}_{j}^{\xi^{2}}=\bigcup_{s=1}^{25}\left\{P_{i j}^{s}\right\} .
$$


All the components $\mathcal{C}_{i}^{\xi}, \mathcal{C}_{i}^{\xi^{2}}$ are embedded with multiplicity 1 in $\overline{\mathcal{S}}$ but $\overline{\mathcal{S}}_{\infty}$ is not reduced, containing embedded points.

Proof. Let $G_{i}(2,4)$ denote the Grassmannian of lines contained in the hyperplane $H_{i}=\left(x_{i}=0\right)$ in $\mathbb{P}^{4}$. By the description of $\overline{\mathcal{S}}$ given above, the reduced structure of $\overline{\mathcal{S}}_{\infty}$ consists of five copies of $L_{i}$, the closure in $G_{i}(2,4)$ of the set

$$
\left\{\{l\} \in G_{i}(2,4) / l \cap B_{i j} \neq \emptyset, l \cap B_{i j k}=\emptyset, \forall j, k \in\{0, \ldots 4\} \backslash\{i\}, j \neq k\right\} .
$$

Take for example $i=4$. Again, $L_{4}$ is a subvariety of the complete intersection curve $C_{4}$ in $\mathrm{G}(2,4)$, which consists of all the lines in $\left(x_{4}=0\right)$ intersecting the curves $B_{4 j}$. $C_{4}$ is given by the following homogeneous ideal

$$
\mathcal{I}=\left(\begin{array}{c}
p_{01}^{5}+p_{02}^{5}+p_{03}^{5}, \\
-p_{01}^{5}+p_{12}^{5}+p_{13}^{5}, \\
-p_{02}^{5}-p_{12}^{5}+p_{23}^{5}, \\
p_{01} p_{23}+p_{03} p_{12}+p_{02} p_{31}
\end{array}\right)
$$

The relation:

$$
\begin{gathered}
5 p_{01} p_{02} p_{03} p_{12} p_{23} p_{31}\left(p_{03} p_{12}+\xi p_{02} p_{13}\right)\left(p_{03} p_{12}+\xi^{2} p_{02} p_{13}\right)= \\
=p_{01}^{5}\left(-p_{02}^{5}-p_{12}^{5}+p_{23}^{5}\right)+p_{12}^{5}\left(p_{01}^{5}+p_{02}^{5}+p_{03}^{5}\right)- \\
-p_{02}^{5}\left(-p_{01}^{5}+p_{12}^{5}+p_{13}^{5}\right)-\left(p_{01}^{5} p_{23}^{5}+\left(p_{03} p_{12}+p_{02} p_{31}\right)^{5}\right)
\end{gathered}
$$

implies the existence of the following irreducible components of $C_{4}$, each appearing with multiplicity 1 :

- 30 curves, corresponding to 30 cones in $\mathbb{P}^{3}$, one through each of the points of $B_{4 i j}, i, j \in\{0, \ldots 3\}$; (recall that each $B_{4 i j}$ is a union of 5 points $B_{4 i j}^{\mu}$, one for each fifth root of unity $\left.\mu=\right)$. One example of the prime ideal of one of these curves would be:

$$
\mathcal{P}_{034}^{\mu}=\left(p_{03}, p_{01}+\mu p_{02}, p_{31}+\mu p_{32},-p_{01}^{5}+p_{12}^{5}+p_{13}^{5}\right)
$$

for $C_{034}^{\mu}$.

- 2 other smooth irreducible curves:

$\mathcal{C}_{4}^{\xi}$ given by the ideal:

$$
\begin{aligned}
& \left(\begin{array}{ccc}
p_{03} p_{12}+\xi p_{02} p_{13}, & p_{01} p_{23}+\xi^{2} p_{02} p_{13}, & \\
p_{01}^{5}+p_{02}^{5}+p_{03}^{5}, & -p_{01}^{5}+p_{12}^{5}+p_{13}^{5}, & -p_{02}^{5}-p_{12}^{5}+p_{23}^{5}
\end{array}\right) \\
& \mathcal{C}_{4}^{\xi^{2}} \text { given by the ideal: } \\
& \left(\begin{array}{ccc}
p_{03} p_{12}+\xi^{2} p_{02} p_{13}, & p_{01} p_{23}+\xi p_{02} p_{13}, & \\
p_{01}^{5}+p_{02}^{5}+p_{03}^{5}, & -p_{01}^{5}+p_{12}^{5}+p_{13}^{5}, & -p_{02}^{5}-p_{12}^{5}+p_{23}^{5}
\end{array}\right)
\end{aligned}
$$

These last two components will be part of $\overline{\mathcal{S}}_{\infty \text { red }}$.

By working with the affine cover of principal open sets $U_{i j}=\left(p_{i j} \neq 0\right)$ of $\mathrm{G}(2,4)$, we can verify that these are all the irreducible components of $C_{4}$, that they are smooth, they appear with multiplicity 1 and 
the multiplicity of intersection of two components at a point of intersection is always 1 . For example, on the affine set $U_{01}$, with coordinates

$$
x_{i}=\frac{-p_{1 i}}{p_{01}}, \quad y_{i}=\frac{p_{0 i}}{p_{01}}
$$

for $i \in\{2,3\}$, the local equations of $C_{4}$ are:

$$
\begin{aligned}
& 1+x_{2}^{5}+x_{3}^{5}=0 \\
& 1+y_{2}^{5}+y_{3}^{5}=0 \\
& x_{2}^{5}-y_{2}^{5}+\left(x_{2} y_{3}-x_{3} y_{2}\right)^{5}=0
\end{aligned}
$$

with

$$
\begin{gathered}
x_{2}^{5}-y_{2}^{5}+\left(x_{2} y_{3}-x_{3} y_{2}\right)^{5}= \\
=x_{2}^{5}\left(1+y_{2}^{5}+y_{3}^{5}\right)-y_{2}^{5}\left(1+x_{2}^{5}+x_{3}^{5}\right)+ \\
+5 x_{2} x_{3} y_{2} y_{3}\left(x_{2} y_{3}+\xi x_{3} y_{2}\right)\left(x_{2} y_{3}+\xi^{2} x_{3} y_{2}\right) .
\end{gathered}
$$

The components of $C_{4}$ intersect at the following points:

- $25 \times 3=75$ points $l_{(i j),(h k)}^{\mu, \nu}$, corresponding to lines passing through points $B_{i j 4}^{\mu}$ and $B_{h k 4}^{\nu}$, with $\{i, j, k, h\}=\{0,1,2,3\}$; each of these $l_{(i j),(h k)}^{\mu, \nu}$ is at the intersection of exactly 2 components: those coming from cones in $\mathbb{P}^{3}$ with vertices $B_{i j 4}^{\mu}, B_{h k 4}^{\nu}$, respectively, over the adequate quintic curves;

- $25 \times 4=100$ points $l_{i j k}^{\mu, \nu}$, corresponding to lines inside the hyperplane $x_{l}=0$, passing through points $B_{i j 4}^{\mu}, B_{i k 4}^{\nu}$ and $B_{j k 4}^{\mu / \nu}$ (here again $\{i, j, k, l\}=\{0,1,2,3\})$. Each of these 100 points lies at the intersection of 5 components of $C_{4}: C_{i j 4}^{\mu}, C_{i k 4}^{\nu}, C_{j k 4}^{\mu / \nu}, \mathcal{C}_{4}^{\xi}$ and $\mathcal{C}_{4}^{\xi^{2}}$.

The above decomposition enables one to compute the Hilbert polynomials $p_{\mathcal{C}_{4}^{\xi}}(n)$ and $p_{S_{\infty}}(n)$ in $G(2,5) \subset \mathbb{P}^{9}$ from successive sequences of the type

$$
\begin{aligned}
& 0 \rightarrow \mathcal{O}_{\mathbb{P}^{9}} /\left(\mathcal{I}_{1} \cap \mathcal{I}_{2}\right) \rightarrow \mathcal{O}_{\mathbb{P}^{9}} / \mathcal{I}_{1} \oplus \mathcal{O}_{\mathbb{P}^{9}} / \mathcal{I}_{2} \rightarrow \mathcal{O}_{\mathbb{P}^{9}} /\left(\mathcal{I}_{1}+\mathcal{I}_{2}\right) \rightarrow 0: \\
& p_{\mathcal{O}_{\mathbb{P}^{9}} / \mathcal{I}_{1}}(n)+p_{\mathcal{O}_{\mathbb{P}^{9}} / \mathcal{I}_{2}}(n)=p_{\mathcal{O}_{\mathbb{P}^{9}} /\left(\mathcal{I}_{1} \cap \mathcal{I}_{2}\right)}(n)+p_{\mathcal{O}_{\mathbb{P}^{9}} /\left(\mathcal{I}_{1}+\mathcal{I}_{2}\right)}(n) .
\end{aligned}
$$

Thus:

$$
\begin{gathered}
\sum_{\{i, j\} \subset\{0,1,2,3\}, \mu} p_{C_{i j 4}^{\mu}}(n)+p_{\mathcal{C}_{4}^{\xi}}(n)+p_{\mathcal{C}_{4}^{\xi^{2}}}(n)=p_{C_{4}}(n)+75+\left(\begin{array}{c}
5 \\
2
\end{array}\right) 100 . \\
p_{C_{i j 4}^{\mu}}(n)=5 n-5,
\end{gathered}
$$

since these curves embed in $\mathbb{P}^{9}$ as plane quintics.

$$
\begin{gathered}
p_{\mathcal{C}_{4}^{\xi}}(n)=p_{\mathcal{C}_{4}^{\xi^{2}}}(n) . \\
p_{C_{4}}(n)=250 n-1375,
\end{gathered}
$$


since $C_{4}$ is a $(5,5,5,2)$ complete intersection in $\mathbb{P}^{5} \subset \mathbb{P}^{9}$. Thus

$$
\begin{gathered}
p_{\mathcal{C}_{4}^{\xi}}(n)=\frac{1}{2}\{250 n-1375+75+1000-30(5 n-5)\}=50 n-75 \\
\overline{\mathcal{S}}_{\infty \text { red }}=\bigcup_{i \in\{0, \ldots 4\}}\left(\mathcal{C}_{i}^{\xi} \bigcup \mathcal{C}_{i}^{\xi^{2}}\right)
\end{gathered}
$$

and each point $l_{i j k}^{\mu, \nu}$ is at the intersection of exactly 4 components $\left(\mathcal{C}_{h}^{\xi}, \mathcal{C}_{h}^{\xi^{2}}\right.$, $\mathcal{C}_{l}^{\xi}$ and $\mathcal{C}_{l}^{\xi^{2}}$, where $\left.\{0, \ldots, 4\} \backslash\{i, j, k, l, h\}\right)$. There are 250 such points when $\{i, j, k\} \subset\{0, \ldots, 4\}$. These points will also be denoted by $\left\{P_{l h}^{s}\right\}_{l \neq h \in\{0, \ldots, 4\}}$, with $s \in\{1, \ldots 25\}$. In our example $h=4$.

Notice also that none of the 375 points $l_{(i j),(h k)}^{\mu, \nu}$ in the base locus is contained in $\overline{\mathcal{S}}_{\infty \text { red }}$. Thus

$$
p_{\overline{\mathcal{S}}_{\infty \text { red }}}(n)=\sum_{i \in\{0, \ldots 4\}} 2 p_{\mathcal{C}_{i}^{\xi}}(n)-\left(\begin{array}{l}
4 \\
2
\end{array}\right) 250=500 n-2250 .
$$

Comparison with the formula for the Hilbert polynomial of the family $\left(\mathcal{S}_{t}\right)_{t}$ in Proposition 4.4 shows that the fiber $\overline{\mathcal{S}}_{\infty}$ must be embedded with multiplicity 1 inside the family $\left(\mathcal{S}_{t}\right)_{t}$. It also shows that there must be some embedded points in $\overline{\mathcal{S}}_{\infty}$, which will give singular points of $\overline{\mathcal{S}}$. We will see shortly that these points are exactly $\left\{P_{l h}^{s}\right\}$, thus each appearing with multiplicity 5 in $\overline{\mathcal{S}}_{\infty}$.

We now proceed with the description of the strict transform $\tilde{\mathcal{S}}$ of $\overline{\mathcal{S}}$ :

Theorem 8.3. The surface $\tilde{\mathcal{S}}$ is smooth.

The fiber $\tilde{\mathcal{S}}_{\infty}$ is the stable limit of the family $\mathcal{S}$ at $t=\infty$ and consists of two connected components $\tilde{\mathcal{C}}^{\xi}$ and $\tilde{\mathcal{C}}^{\xi^{2}}$, which are isomorphic and reducible:

$$
\tilde{\mathcal{C}}^{\xi}=\bigcup_{i=0}^{4} \tilde{\mathcal{C}}_{i}^{\xi}
$$

with $\tilde{\mathcal{C}}_{i}^{\xi}$ smooth isomorphic curves of genus 76 , intersecting pairwise transversely at 25 points each.

Proof. By examining the morphism $p: \tilde{G}(2,5) \rightarrow G(2,5)$ locally, we will see that no fibers of the exceptional divisors are contained in $\tilde{\mathcal{S}}$, that for each pair $\mathcal{C}_{i}^{\xi}$ and $\mathcal{C}_{i}^{\xi^{2}}$, the two components are separated and $p$ desingularizes $\overline{\mathcal{S}}$.

We will work over $\mathbb{A}^{6} \cong U_{01} \in G(2,5)$. Looking at the restriction of $p$ :

$$
\left(B l_{(0,0)} \mathbb{A}^{2}\right)^{3} \longrightarrow \mathbb{A}^{6}
$$

one finds two types of affine charts, which after all possible permutations cover the entire $\tilde{V}_{01}$ : 
(1) $\mathbb{A}^{6}$ with coordinates $\left\{u_{i}, y_{i}\right\}_{i \in\{2,3,4\}}$, where the original coordinates $\left\{x_{i}, y_{i}\right\}_{i \in\{2,3,4\}}$ on $U_{01}$ satisfy:

$$
x_{i}=u_{i} y_{i}
$$

The exceptional divisor $E_{i}=\left(y_{i}=0\right)$ and $u_{i}$ is the coordinate along the fiber of the exceptional divisor over the corresponding component of the blow-up locus.

(2) $\mathbb{A}^{6}$ with coordinates $\left\{u_{2}, y_{2}, u_{3}, y_{3}, x_{4}, v_{4}\right\}$.

$$
x_{2}=u_{2} y_{2}, x_{3}=u_{3} y_{3}, y_{4}=x_{4} v_{4} .
$$

$\left(y_{2}\right),\left(y_{3}\right)$ and $\left(x_{4}\right)$ give the exceptional divisors.

Case (1) is closely related to the setup of Proposition 4.1. Indeed, in this local picture formula (4.1) becomes

$$
\phi(\alpha: \beta)=\left(\alpha: \beta: y_{2}\left(u_{2} \alpha+\beta\right): y_{3}\left(u_{3} \alpha+\beta\right): y_{4}\left(u_{4} \alpha+\beta\right)\right)
$$

and thus by the same reasoning as in Proposition 4.1:

$$
\left(\begin{array}{lll}
u_{2} & u_{3} & u_{4} \\
u_{2}^{2} & u_{3}^{2} & u_{4}^{2} \\
u_{2}^{3} & u_{3}^{3} & u_{4}^{3} \\
u_{2}^{4} & u_{3}^{4} & u_{4}^{4}
\end{array}\right)\left(\begin{array}{c}
y_{2}^{5} \\
y_{3}^{5} \\
y_{4}^{5}
\end{array}\right)=5 t \prod_{j=2}^{4} y_{j}\left(\begin{array}{c}
\frac{1}{5} s_{0}(u) \\
\frac{1}{10} s_{1}(u) \\
\frac{1}{10} s_{2}(u) \\
\frac{1}{5} s_{3}(u)
\end{array}\right)
$$

plus the separate equations

$$
\sigma_{5}+1=\sigma_{0}+1=0,
$$

where the notations are the same as in section 4 , except that in this case

$$
u=\left(u_{2}: u_{3}: u_{4}\right)
$$

As before, one sees that the fourth row of the augmented matrix is just a linear combination of the others. After removing the exceptional divisors $E_{2}, E_{3}, E_{4}$, one is left with the irreducible surface $\tilde{\mathcal{S}}$ given by:

$$
\sigma_{5}+1=\sigma_{0}+1=\sigma_{2}-\frac{1}{2} s_{1} \sigma_{1}=\sigma_{3}-\frac{1}{2} s_{2} \sigma_{1}=0 .
$$

Consider the intersection with the exceptional divisor $E_{4}$ : Then $y_{4}=0$ and $\sigma_{1}, \sigma_{2}, \sigma_{3}$ satisfy in this case the identity:

$$
\sigma_{3}-\left(u_{2}+u_{3}\right) \sigma_{2}+u_{2} u_{3} \sigma_{1}=0
$$

which in terms of the equations (8.1) is equivalent to:

$$
\sigma_{1}\left(u_{2}^{2}-u_{2} u_{3}+u_{3}^{2}\right)=0
$$

The conditions $u_{2}=-\xi u_{3}$ and $u_{2}=-\xi^{2} u_{3}$, where $\xi^{2}+\xi+1=0$, give the direct transforms $\tilde{\mathcal{C}}_{4}^{\xi}$ and $\tilde{\mathcal{C}}_{4}^{\xi^{2}}$ of $\mathcal{C}_{4}^{\xi}$ and $\mathcal{C}_{4}^{\xi^{2}}$. Notice that in this open set $\tilde{\mathcal{C}}_{4}^{\xi}$ and $\tilde{\mathcal{C}}_{4}^{\xi^{2}}$ do not intersect, because condition $\sigma_{5}+1=0$ excludes the case $u_{2}=u_{3}=0$. Also, in this open set $\tilde{\mathcal{S}}$ contains no fibers in the exceptional divisor $E_{4}$ : such fibers would satisfy $\sigma_{1}=0$, which would also imply $\sigma_{2}=0$. But these two equations in $u_{2}, u_{3}, y_{2}, y_{3}$ have no common solutions with $\sigma_{5}+1=\sigma_{0}+1=0$. 
The intersection $\tilde{\mathcal{S}} \cap E_{4} \cap E_{3}$ immediately gives:

$$
y_{3}=y_{4}=0, \quad y_{2}=-\mu, \quad u_{2}=-\nu
$$

for two fifth roots of unity $\mu$ and $\nu$. Also, $\sigma_{2}-\frac{1}{2} s_{1}=0$ becomes

$$
u_{2}-u_{3}-u_{4}=0 \text {, }
$$

so in view of equations (8.1) and (8.2), one sees that $\tilde{\mathcal{C}}_{3}^{\xi}$ intersects $\tilde{\mathcal{C}}_{4}^{\xi}$ at 25 points given by

$$
y_{3}=y_{4}=0, \quad y_{2}=-\mu, \quad u_{2}=-\nu, \quad u_{3}=\xi^{2} \nu, \quad u_{4}=\xi \nu
$$

and $\tilde{\mathcal{C}}_{3}^{\xi^{2}}$ intersects $\tilde{\mathcal{C}}_{4}^{\xi^{2}}$ at 25 points given by

$$
y_{3}=y_{4}=0, \quad y_{2}=-\mu, \quad u_{2}=-\nu, \quad u_{3}=\xi \nu, \quad u_{4}=\xi^{2} \nu
$$

and these are all the points in $\tilde{\mathcal{S}} \cap E_{4} \cap E_{3}$.

In the above, $\tilde{\mathcal{C}}_{4}^{\xi}$ and $\tilde{\mathcal{C}}_{4}^{\xi}$ have been fixed; after permutations, the appropriate choices of notation for $\tilde{\mathcal{C}}_{i}^{\xi}$ and $\tilde{\mathcal{C}}_{i}^{\xi^{2}}$ can be made, by keeping track of the signatures of the permutations. In this way the set of components of $\tilde{\mathcal{S}}_{\infty}$ found thus far splits in two subsets $\left\{\tilde{\mathcal{C}}_{i}^{\xi}\right\}_{i}$ and $\left\{\tilde{\mathcal{C}}_{i}^{\xi^{2}}\right\}_{i}$, such that all the curves in one subset intersect pairwise transversely, but do not intersect the components in the other subset. The smoothness of $\tilde{\mathcal{S}}$ along the points of $\tilde{\mathcal{S}}_{\infty}$ is easily checked by differentiating equations (8.2) and using the conditions derived from (8.3).

The only thing that remains to be checked is existence of fibers in the exceptional divisors, or intersection points for $\tilde{\mathcal{C}}_{i}^{\xi}$ and $\tilde{\mathcal{C}}_{i}^{\xi^{2}}$ in sets of type (2). Here equations (8.2) are written for

$$
\begin{gathered}
\sigma_{i}=u_{2}^{i} y_{2}^{5}+u_{3}^{i} y_{3}^{5}+x_{4}^{5} v_{4}^{i}, \\
s_{0}=v_{4}, s_{1}=\left(u_{3}+u_{3}\right) v_{4}+1, s_{2}=u_{2} u_{3} v_{4}+u_{2}+u_{3}, s_{3}=u_{2} u_{3} .
\end{gathered}
$$

A simple check following the lines of the previous computations shows that there are no extra intersection points, nor fibers in the exceptional divisors.

The genus computation for $\tilde{\mathcal{S}}_{\infty}$ gives exactly:

$$
1250=2 \cdot(5 \cdot 75+250)
$$

Theorem 8.4. The action of the group $\hat{G}$ on $G(2,5)$ extends canonically to an action on $\tilde{G}(2,5)$, making the morphism

$$
p: \tilde{G}(2,5) \rightarrow G(2,5)
$$

$\hat{G}$-equivariant. $\tilde{\mathcal{S}} / \hat{G}$ is a smooth surface. The stable limit at $w=\infty$ of the family $\mathcal{S} / \hat{G}$ is

$$
\tilde{\mathcal{S}}_{\infty} / \hat{G}=\left(\tilde{\mathcal{C}}^{\xi} / \hat{G}\right) \bigcup\left(\tilde{\mathcal{C}}^{\xi^{2}} / \hat{G}\right)
$$

where $\tilde{\mathcal{C}}^{\xi} / \hat{G}$ is the union of 5 smooth rational curves intersecting pairwise transversely. 
Proof. Working locally over $\mathbb{A}^{6} \cong U_{01} \in G(2,5)$, and considering for $\tilde{G}(2,5)$ the local coordiantes set up in the proof of Theorem 8.3, it is not difficult to derive the action of $\hat{G}$ on $\tilde{G}(2,5)$ : If

$$
g=\left(1: \mu_{1}: \mu_{2}: \mu_{3}: \mu_{4}\right) \in \hat{G}
$$

and $\{l\} \in G(2,5)$ is the line passing through $\left(1: 0: x_{2}: x_{3}: x_{4}\right),\left(0: 1: y_{2}:\right.$ $\left.y_{3}: y_{4}\right)$, then

$$
\begin{gathered}
g^{*}\left(x_{i}\right)=\mu_{i} x_{i}, \\
g^{*}\left(y_{i}\right)=\mu_{i} y_{i} / \mu_{1},
\end{gathered}
$$

and since $x_{i}=u_{i} y_{i}$, it follows that

$$
g^{*}\left(u_{i}\right)=\mu_{1} u_{i}
$$

for all $i \in\{2,3,4\}$.

Recall from Theorem 5.2 that $\mathcal{S} / \hat{G}$ is smooth. The proof that all points of $\tilde{\mathcal{S}}_{\infty} / \hat{G}$ are smooth in $\tilde{\mathcal{S}} / \hat{G}$ follows the same argument as in the proof of Theorem 5.2. A general point of $\tilde{\mathcal{C}}_{4}^{\xi}$, for example, has an order 5 isotropy group, generated by an element of the form $(1: 1: 1: 1: \mu)$, with $\mu^{5}=1$. A point in $\tilde{\mathcal{C}}_{4}^{\xi} \cap \tilde{\mathcal{C}}_{3}^{\xi}$ has an order 25 isotropy group, generated by two elements of the form $(1: 1: 1: 1: \mu),(1: 1: 1: \mu: 1)$, with $\mu^{5}=1$. Each of these elements of $g$ gives a pseudoreflection of $\tilde{\mathcal{S}}$, because the set of points in $\tilde{\mathcal{S}}$ fixed by it is the divisor $\tilde{\mathcal{C}}_{4}^{\xi} \cup \tilde{\mathcal{C}}_{4}^{\xi^{2}}, \tilde{\mathcal{C}}_{3}^{\xi} \cup \tilde{\mathcal{C}}_{3}^{\xi^{2}}$ respectively. Again following $\underline{\mathrm{ST}}$, $\tilde{\mathcal{S}} / \hat{G}$ is smooth.

The Hurwitz formula for the genus $g^{\prime}$ of $\tilde{\mathcal{C}}_{4}^{\xi} / \hat{G}$ :

$$
2(76-1)=125 \cdot\left(g^{\prime}-1\right)+100 \cdot 4
$$

implies $g^{\prime}=0$. The 25 points of $\tilde{\mathcal{C}}_{4}^{\xi} \cap \tilde{\mathcal{C}}_{3}^{\xi}$ are all in the same orbit, thus giving one point in the quotient. Notice that the above numbers sum up to a total genus of 6 for $\tilde{\mathcal{C}}^{\xi} / \hat{G}$.

\section{The CLASS $[\overline{\mathcal{S}}]$ ON $G(2,5)$}

Let $F(1,2,5)$ denote the flag variety

$$
F(1,2,5)=\{(x,[l]) /[l] \in G(2,5), x \in l\}
$$

with the natural morphisms:

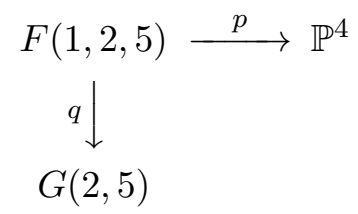


and consider the induced diagram

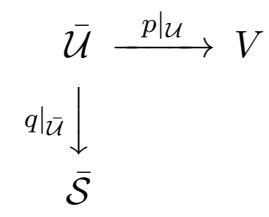

where $\overline{\mathcal{U}}$ is the pull-back of $F(1,2,5)$ on $\overline{\mathcal{S}}$ and $V=p(\mathcal{U})$.

Here we will use the incidence variety $I$ and the description of its components from Section 8 to compute the class $[\overline{\mathcal{S}}]$ of $\overline{\mathcal{S}}$ in the cohomology ring of the Grassmannian, and the degree of $V$ as threefold in $\mathbb{P}^{4}$. The method is to compute the class of each component of $I$ and then eliminate the irrelevant components to get $\overline{\mathcal{S}}$. Recall that $I$ comprises:

(1) 50 components, each of which consists of lines in a cone over one of the $B_{l}$-s and having as vertex one of the points in $B_{i j k}$,

(2) 15 components, each of which consists of lines intersecting two of the curves $B_{i j}$ and $B_{k l}$,

(3) 10 components, each of which consists of lines intersecting one $B_{i j}$ and $B_{k}$ and $B_{l}$ and $B_{h}$ (but in general not $B_{k l}, B_{k h}$ or $B_{l h}$, nor $B_{i j k} B_{i j l}$ or $\left.B_{i j h}\right),(4) \overline{\mathcal{S}}$.

Lemma 9.1. Let I be given in the Grassmannian $G(2,5)$ by the ideal

$$
\mathcal{I}=\left(\sum_{j=0}^{4} p_{i j}^{5}\right)_{i \in\{1, \ldots, 4\}} .
$$

Then all the 2-dimensional components of I appear with multiplicity 1 in the ideal $\mathcal{I}$.

Proof. The proof of Proposition 8.1 implies the present lemma for those components of $I$ contained in one of the Grassmannians $G_{i}(2,4)$. In particular, $\overline{\mathcal{S}}$ appears with multiplicity 1 . Consider now the ideal $\mathcal{I}$ localized on $U_{01}=\left(p_{01} \neq 0\right)$, with the usual coordinates $\left\{x_{i}, y_{i}\right\}_{i \in\{2,3,4\}}$. The generators of $\mathcal{I}$ :

$$
\begin{gathered}
f_{1}=1+x_{2}^{5}+x_{3}^{5}+x_{4}^{5}, \\
f_{2}=1+y_{2}^{5}+y_{3}^{5}+y_{4}^{5}, \\
f_{3}=x_{3}^{5}-y_{3}^{5}+\left(x_{3} y_{2}-x_{2} y_{3}\right)^{5}+\left(x_{3} y_{4}-x_{4} y_{3}\right)^{5}, \\
f_{4}=x_{4}^{5} y_{0}^{5}-x_{1}^{5} y_{4}^{5}+\left(x_{4} y_{2}-x_{2} y_{4}\right)^{5}+\left(x_{4} y_{3}-x_{3} y_{4}\right)^{5} .
\end{gathered}
$$

satisfy the following relations:

$$
\begin{aligned}
& f_{3}=5 x_{3} y_{3} g_{3}+x_{3}^{5} f_{2}-y_{3}^{5} f_{1} \\
& f_{4}=5 x_{4} y_{4} g_{4}+x_{4}^{5} f_{2}-y_{4}^{5} f_{1}
\end{aligned}
$$

where for $\{i, j, k\}=\{2,3,4\}$,

$$
\begin{gathered}
g_{j}=p_{i j} p_{i j}^{\xi} p_{i j}^{\xi^{2}} x_{i} y_{i}-p_{j k} p_{j k}^{\xi} p_{j k}^{\xi^{2}} x_{k} y_{k} \\
p_{i j}=x_{i} y_{j}-x_{j} y_{i}, \quad p_{i j}^{\xi}=x_{i} y_{j}+\xi x_{j} y_{i}, \quad p_{i j}^{\xi^{-1}}=x_{i} y_{j}+\xi^{-1} x_{j} y_{i}
\end{gathered}
$$


DEGREE 1 CURVES IN THE DWORK PENCIL AND THE MIRROR QUINTIC 41

with $\xi^{2}+\xi+1=0$. Relations 9.1 and 9.2 highlight the existence of 2 dimensional components of the type:

$$
\begin{gathered}
\left(x_{i}, y_{j}, f_{1}, f_{2}\right), \quad\left(x_{i}, x_{j}, f_{1}, f_{2}\right), \quad\left(y_{i}, y_{j}, f_{1}, f_{2}\right), \\
\left(p_{i j}, p_{j k}, p_{k i}, f_{1}, f_{2}\right), \quad\left(p_{i j}, p_{j k}^{\xi}, p_{k i}^{\xi^{2}}, f_{1}, f_{2}\right)
\end{gathered}
$$

for $\{i, j, k\}=\{2,3,4\}$ A straighforward exercise shows that these components also appear with multiplicity one and they cover all types (1)-(3) of components written above.

For computations in the cohomology ring of $G(2,5)$, we will use the following Schubert cycles: $\sigma_{\left(a_{1}, a_{2}\right)}$ is the class of $\left\{l \mid \operatorname{dim}\left(l \cap V_{3-a_{i}+i} \geq i\right\}\right.$, for a fixed flag $V_{1} \subset V_{2} \subset \mathbb{P}^{4}$.

\section{Proposition 9.2.}

$$
[\overline{\mathcal{S}}]=5^{3} \cdot 3 \sigma_{22}+5^{3} \cdot 2 \sigma_{31} .
$$

Proof. The different types of components of $I$ mentioned above give the following cycles:

(0) $[I]$ corresponds to the cycle of lines intersecting 4 quintic surfaces in $\mathbb{P}^{4}$ :

$$
[I]=5^{4} \sigma_{1}^{4}=5^{4} \cdot 2 \sigma_{22}+5^{4} \cdot 3 ; \sigma_{31}
$$

(1) $\left[I_{1}\right]$ corresponds to the cycle of lines contained in a cone over a quintic surface:

$$
\left[I_{1}\right]=5 \sigma_{31}
$$

(2) $\left[I_{2}\right]$ corresponds to the cycle of lines intersecting two plane quintic curves:

$$
\left[I_{2}\right]=25 \sigma_{20}^{2}=25\left(\sigma_{22}+\sigma_{31}\right) ;
$$

(3) the cycle of lines intersecting a degree 5 plane curve and two degree 5 surfaces consists of $\left[I_{3}\right]$ together with 3 copies of $\left[I_{2}\right]$ and 15 copies $\left[I_{1}\right]$ (again, coming from the geometry of the base locus $B$ ). Thus:

$$
\begin{aligned}
{\left[I_{3}\right] } & =125 \sigma_{10}^{2} \cdot \sigma_{20}-3 \cdot 25\left(\sigma_{22}+\sigma_{31}\right)-15 \cdot 5 \sigma_{31} \\
& =125\left(\sigma_{11}+\sigma_{20}\right) \sigma_{20}-75\left(\sigma_{22}+\sigma_{31}\right)-75 \sigma_{31}= \\
& =125\left(2 \sigma_{31}+\sigma_{22}\right)-75\left(\sigma_{22}+\sigma_{31}\right)-75 \sigma_{31}= \\
& =100 \sigma_{31}+50 \sigma_{22}
\end{aligned}
$$

Finally,

$$
[\overline{\mathcal{S}}]=[I]-50\left[I_{1}\right]-15\left[I_{2}\right]-10\left[I_{3}\right]=5^{3} \cdot 3 \sigma_{22}+5^{3} \cdot 2 \sigma_{31} .
$$

The degree of the surface $\overline{\mathcal{S}}$ in $\mathbb{P}^{9}$ is thus:

$$
\operatorname{deg}(\overline{\mathcal{S}})=\int_{G(2,5)} \sigma_{1}^{2} \cdot[\overline{\mathcal{S}}]=5^{4} .
$$

Proposition 9.3. The locus $V$ covered by all the lines inside the Dwork pencil is a degree 250 threefold in $\mathbb{P}^{4}$. 
Proof. Recall the morphisms

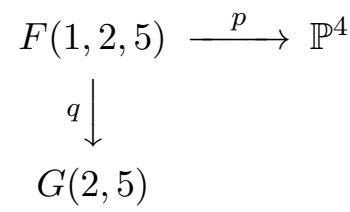

Let $H=p^{*} \mathcal{O}_{\mathbb{P}^{4}}(1)$. The degree of $V$ is:

$$
\operatorname{deg}(V)=\int_{F(1,2,5)} q^{*}[\overline{\mathcal{S}}] \cdot H^{3} .
$$

By projection formula and the identity $q_{*}\left(H^{3}\right)=\sigma_{2}$ :

$$
\operatorname{deg}(V)=\left(5^{3} \cdot 3 \sigma_{22}+5^{3} \cdot 2 \sigma_{31}\right) \cdot \sigma_{2}=250 .
$$

Proposition 9.4. For each $i \in\{0, \ldots, 4\}$, there is a finite, 30-1 morphism

$$
\tilde{\mathcal{S}} \longrightarrow B_{i}
$$

having 1-dimensional fibers over points of the type

$$
(1:-1: 0: 0: 0), \quad\left(1: \xi: \xi^{2}: 0: 0\right)
$$

and their orbits through the actions of $\hat{G}$ and of the group of symmetries $S_{5}$, and branched over points of type

$$
\left(x_{0}: x_{1}: x_{2}: 0: 0\right)
$$

with $x_{0}^{5}+x_{1}^{5}+x_{2}^{5}=0$ (and their orbits through the actions of $\hat{G}$ and $S_{5}$ ).

Proof. There is a well defined morphism from the blow-up of $G(2,5)$ along $G_{i}(2,4)$ to the hyperplane $H_{i}$ in $\mathbb{P}^{4}$ :if we think of this blow-up as sitting inside $F(1,2,5)$, then the morphism sends the pair $(x,[l]) \in F(1,2,5)$ to $l \cap H_{i}$ if $l$ is not contained in $H_{i}$, or to $x$ if $l \subset H_{i}$. The image of the strict transform of $\overline{\mathcal{S}}$ through this morphism is exactly the Fermat surface $B_{i}$. Hence the morphism

$$
\tilde{\mathcal{S}} \stackrel{\varphi_{i}}{\longrightarrow} B_{i} .
$$

To compute the degree of $\varphi_{i}$, set $i=0$ and consider a generic point $x$ of $B_{0}$. Consider the projection of vertex $x$ down to $H_{4}$ and count the points where the images of the surfaces $B_{1}$ and $B_{2}$ intersect $B_{4}$. There are $125 \mathrm{such}$ points: the projections of the 125 lines having contact with each of $B_{0}, B_{1}$, $B_{2}$ and $B_{4}$ and therefore with $B_{3}$ also. Now we count how many of these lines are in components of type (1)-(3) of $I$ : the 6 curves $B_{i j}$ with $i, j \neq 0$ give 150 lines through $x$, that intersect $B_{i j}$ and the other $B_{k}$-s. However, the lines through the 20 points of $B_{i j k},(i, j, k \neq 0)$, have been counted three times in this process. By adjusting: $125-150+2 \cdot 20=15$ lines.

There is another special set of lines, which have been counted twice: lines through $x$ and intersecting two curves $B_{i j}$ and $B_{k l}$ with $\{i, j, k, l\}=$ $\{1,2,3,4\}$. Indeed, lines intersecting $B_{i j}$ and $B_{k l}$ automatically intersect $B_{0}$ 
and by dimension count, the points of intersection with $B_{0}$ of all such lines cover the whole $B_{0}$. For each choice of pairs $(i, j)$ and $(k, l)$, there are 5 such lines passing trough $x$, since the projections of the planes $\left(x_{i}=x_{j}=0\right)$ and $\left(x_{k}=x_{l}=0\right)$ to the hyperplane $H_{4}$ intersect each other and $B_{4}$ in 5 points. A total of 15 lines has thus been counted twice. Adding up, one obtains 30 lines through $x$ which are in the universal family $\mathcal{U}$.

The special fibers of the morphism $\varphi_{i}$ occur at the special lines studied: those in $\mathcal{S}_{0}$ and the Van Geemen lines.

Let $V_{t}=p_{*} q^{*}\left(\overline{\mathcal{S}}_{t}\right)$, for any $t \in \mathbb{P}^{1} . V_{t}$ is a degree 500 surface in $\mathbb{P}^{4}$, as can be easily checked either at $t=0$ or $t=\infty$.

Notice that by our computations, $V \cap X_{t}=V_{t} \cup\left(\bigcup_{i=0}^{4} B_{i}\right)$ is a reduced surface of degree $250 \cdot 5=1250$. As expected, each of the 5 surfaces $B_{i}$ appears with multiplicity 30 in $V \cap X_{t}$ :

$$
1250=500+30 \cdot 5 \cdot 5
$$

Remark 9.5. In unpublished work, G.Pacienza has studied the

2-dimensional locus of points where lines intersect a generic quintic threefold $X$ with multiplicity at least 5 . He has shown that this locus has degree 650 . If $X=X_{t}$ is one of the quintics in the Dwork pencil, one can now state that the above locus is reducible to the degree 500 surface $V_{t}$ and the 5 components of the base locus of $\mathcal{X}$, each appearing with multiplicity 6 . This is also in agreement with the numerical results of section 9.

\section{REFERENCES}

[AK1] A. Albano, S. Katz, Lines on the Fermat Quintic Threefold and the Infinitesimal Generalized Hodge Conjecture, Transactions of the AMS. 324 (1991), no.1, 353-368.

[AK2] A. Albano, S. Katz, Van Geemen's Families of Lines on Special Quintic Threefolds. Manuscripta Math. 70 (1991), no. 2, 183-188.

[C1] H. Clemens, Cohomology and Obstructions I: On the geometry of formal Kuranishi theory, arXiv: math.AG/9901084.

[C2] H. Clemens, Cohomology and Obstructions II: Curves on K-Trivial Threefolds arXiv: math.AG/0206219

[CK] H. Clemens, H. P. Kley Counting curves which move with threefolds, J. Algebraic Geometry 9 (2000), no. 1, 175-200.

[F] William Fulton, Intersection Theory, Springer-Verlag:1984

[GH] P.Griffiths, J.Harris, Principles of Algebraic Geometry, Wiley-Interscience, New York, 1978

[K] J. Kollár, Rational Curves on Algebraic Varieties, Ergebnisse der Mathematik und Grenzgebiete 3. Folge, vol.32, Springer-Verlag:1996

[ST] G. S. Shephard, Finite Reflection Groups, Canadian J. Math. 6 (1954), 274-304;

Department of Mathematics, University of British Columbia, Room 121, 1984 Mathematics Road, Vancouver, B.C., Canada, V6T1Z2

E-mail address: amustata@math.ubc.edu 